\title{
$K$-Theory and Geometric Topology
}

\author{
Jonathan Rosenberg* \\ University of Maryland, College Park, MD, 20742, USA, jmr@math.umd.edu
}

Historically, one of the earliest motivations for the development of $K$-theory was the need to put on a firm algebraic foundation a number of invariants or obstructions that appear in topology. The primary purpose of this chapter is to examine many of these $K$-theoretic invariants, not from a historical point of view, but rather a posteriori, now that $K$-theory is a mature subject.

There are two reasons why this may be a useful exercise. First, it may help to show $K$-theorists brought up in the "algebraic school" how their subject is related to topology. And secondly, clarifying the relationship between $K$ theory and topology may help topologists to extract from the wide body of $K$-theoretic literature the things they need to know to solve geometric problems.

For purposes of this article, "geometric topology" will mean the study of the topology of manifolds and manifold-like spaces, of simplicial and CWcomplexes, and of automorphisms of such objects. As such, it is a vast subject, and so it will be impossible to survey everything that might relate this subject to $K$-theory. I instead hope to hit enough of the interesting areas to give the reader a bit of a feel for the subject, and the desire to go off and explore more of the literature.

Unless stated otherwise, all topological spaces will be assumed to be Hausdorff and compactly generated. (A Hausdorff space $X$ is compactly generated if a subset $C$ is closed if and only if $C \cap K$ is closed, or equivalently, compact, for all compact subsets $K$ of $X$. Sometimes compactly generated spaces are called $k$-spaces. The $k$ stands both for the German Kompakt and for Kelley, who pointed out the advantages of these spaces.) This eliminates certain pathologies that cause trouble for the foundations of homotopy theory. "Map" will always mean "continuous map." A map $f: X \rightarrow Y$ is called a weak equivalence if its image meets every path component of $Y$ and if $f_{*}: \pi_{n}(X, x) \rightarrow \pi_{n}(Y, f(x))$ is an isomorphism for every $x \in X$.

\footnotetext{
* Partially supported by NSF Grant DMS-0103647. The author thanks John Rognes and Allen Hatcher for helpful comments on the first draft of this article.
} 


\section{The Wall Finiteness Obstruction and its Variants}

We begin this survey with the "Wall finiteness obstruction," not because it came first historically (Whitehead torsion dates back much earlier) and not because it is most important (again, most geometric topologists would argue that Whitehead torsion is more fundamental) but because most algebraic treatments of $K$-theory usually begin with $K_{0}$ of a ring or a category.

The discussion here will be brief; for a more complete treatment, see [33].

A basic theorem of homotopy theory states that every space $X$ has a $C W$-approximation; in other words, there is a $\mathrm{CW}$-complex $Y$ and a weak equivalence $Y \rightarrow X$. More is true; the $Y$ is unique up to homotopy equivalence and can be chosen functorially in $X$. In fact one can take $Y=\left|S_{\bullet}(X)\right|$ to be the geometric realization of the simplicial set $S_{\bullet}(X)$ of singular $n$-simplices in $X[59$, Chaps. 10, 16].

One says a space $X$ is dominated by a space $Y$ if there are maps $f: X \rightarrow Y$ and $g: Y \rightarrow X$ such that $g \circ f \simeq 1_{X}$, where the symbol $\simeq$ denotes "is homotopic to." A corollary of the CW-approximation theorem is that if a space $X$ is dominated by a CW-complex, then it is homotopy-equivalent to a CW-complex. We say $X$ is finitely dominated if it is dominated by a finite $\mathrm{CW}$-complex. Clearly this is a necessary condition for $X$ to be homotopyequivalent to a finite $\mathrm{CW}$-complex. The condition of being finitely dominated is sometimes not so hard to check. For example, a famous theorem of Borsuk [9, p. 1093] implies that any compact, locally contractible, and finite dimensional metric space is a retract of a finite polyhedron, hence in particular is finitely dominated.

Theorem 1.1 (Wall [100], [101]). Let $X$ be a path-connected and locally 1-connected space, and let $C_{*}(X)$ be its singular chain complex. Note that the singular chain complex $C_{*}(\widetilde{X})$ of the universal cover $\widetilde{X}$ can be regarded as a complex of free $R$-modules, where $R=\mathbb{Z} \pi_{1}(X)$, and that $C_{*}(X)=\mathbb{Z} \otimes_{R} C_{*}(\widetilde{X})$. Then if $X$ is finitely dominated, $\pi_{1}(X)$ is finitely presented and $C_{*}(\widetilde{X})$ is chain homotopy-equivalent to a finite complex $C_{*}$ of finitely generated projective R-modules. The "Euler characteristic" of this complex,

$$
\chi(X)=\sum_{i}(-1)^{i}\left[C_{i}\right],
$$

is well defined in $\widetilde{K}_{0}(R)$ (the quotient of $K_{0}(R)$ by the copy of $\mathbb{Z}$ coming from the finitely generated free $R$-modules), and vanishing of $\chi(X)$ in $\widetilde{K}_{0}(R)$ is necessary and sufficient for $X$ to be homotopically finite (homotopy-equivalent to a finite $C W$-complex.)

Proof. We give a brief sketch. If $X$ is finitely dominated, then $\pi_{1}(X)$ is an algebraic retract of a finitely presented group, hence is itself finitely presented. First we note that the Euler characteristic $\chi(X)$ is well defined. The key thing to prove is that if there is a chain equivalence $h: C_{*} \rightarrow C_{*}^{\prime}$, then 
$\sum_{i}(-1)^{i}\left[C_{i}\right]=\sum_{i}(-1)^{i}\left[C_{i}^{\prime}\right]$. But this is true even for the $K_{0}(R)$-valued Euler characteristic (not only for its image in $\widetilde{K}_{0}(R)$ ), by the Euler-Poincaré principle.

Clearly, if there is an equivalence $Z \rightarrow X$ with $Z$ a finite CW-complex, then $C_{*}(X)$ is chain homotopy-equivalent to $C_{*}(\widetilde{Z})$, which in dimension $j$ is a free $R$-module with one generator for each $j$-cell in $Z$. Thus $\left[C_{i}(\widetilde{Z})\right]$ lies in the subgroup $\mathbb{Z}$ of $K_{0}(R)$ generated by the free modules, and maps to 0 in $\widetilde{K}_{0}(R)$, so $\chi(X)=\chi(Z)=0$.

Wall's main contribution was to prove sufficiency of the condition. First one shows that if $\chi(X)=0$, then $C_{*}(\widetilde{X})$ is chain equivalent to a finite complex of finitely generated free $R$-modules. This is elementary; start with an equivalent finite complex $C_{*}$ of projective modules, say of dimension $n$, and choose a finitely generated projective $R$-module $Q_{0}$ such that $C_{0} \oplus Q_{0}$ is free. Then the direct sum of $C_{*}$ with the complex

$$
Q_{0} \cong Q_{0} \leftarrow 0 \leftarrow \cdots
$$

is still equivalent to $C_{*}(\widetilde{X})$ and is free in degree 0 . Proceed similarly by induction. Since $\chi(X)=0$, once the $(n-1)$-st module has been made free, the $n$-th module is stably free. So making $Q_{n-1}$ larger if necessary, one can arrange that all the modules are now free (and still finitely generated).

The last step is to build a finite CW-complex $Z$ modeling the free chain complex from the last step, and to construct the required homotopy equivalence $h$. The $Z$ and the $h$ are constructed simultaneously by starting with a 2-complex $Z^{(2)}$ with the correct fundamental group (recall $\pi_{1}(X)$ is finitely presented) and with the correct $C_{1}$ and $C_{2}$, along with a map $h^{(2)}: Z^{(2)} \rightarrow X$ inducing an isomorphism on $\pi_{1}$. Then one attaches cells and extends the map by induction on the dimension. This is an exercise in obstruction theory. Eventually one gets the desired complex $Z$ and a map $h: Z \rightarrow X$ which is an isomorphism on $\pi_{1}$ and which induces a homology isomorphism $\widetilde{Z} \rightarrow \widetilde{X}$. By Whitehead's Theorem, this map is a homotopy equivalence.

One situation where the Wall finiteness obstruction comes into play is the spherical space form problem. This is the problem of determining what finite groups $G$ can act freely on $S^{n}$. Of course, there are certain obvious examples, namely groups which act freely and isometrically on $S^{n}$ with its standard metric. These are classified in [105]. The necessary and sufficient condition for $G$ to act freely and isometrically on some $S^{n}$ is that for all primes $p$ and $q$, not necessarily distinct, all subgroups of $G$ of order $p q$ must be cyclic. But if one doesn't require the action to be isometric (or even smooth), there are many more examples. The one obvious necessary condition is a homological one. For if $X$ is a connected CW-complex with finite fundamental group $G$ and with universal cover $\widetilde{X}$ homotopy-equivalent to $S^{n}$, then the spectral sequence

$$
H^{p}\left(G, H^{q}(\tilde{X}, \mathbb{Z})\right) \Rightarrow H^{p+q}(X, \mathbb{Z})
$$


of the homotopy fibration $\widetilde{X} \rightarrow X \rightarrow B G$ implies that $G$ has periodic cohomology of period $n+1$, and thus that the Sylow subgroups of $G$ are all either cyclic or generalized quaternion [17, Ch. XVI, $\S 9$, Application 4]. Conversely, if $G$ satisfies this condition, Swan [87] showed that there is a periodic resolution of the trivial $G$-module $\mathbb{Z}$ by finitely generated projective $\mathbb{Z} G$-modules. In effect, the finiteness obstruction of this resolution is an obstruction to $G$ acting freely and cellularly on a finite homotopy $n$-sphere. (We are explaining this a posteriori; Swan's paper predated Wall's, but the principle is the same.) But since $\widetilde{K}_{0}(\mathbb{Z} G)$ is finite for $G$ finite, one can kill off the obstruction by replacing the period by a suitably large multiple. Thus the result of [87] is that, after replacing the period of $G$ by a suitably large multiple if necessary, $G$ acts freely and cellularly on a finite $n$-dimensional $\mathrm{CW}$-complex complex $\widetilde{X}$ homotopy-equivalent to $S^{n}, n$ one less than this larger period. For an explanation of how one then checks if $X$ can be chosen to be a smooth manifold, see [88], [58], and [26]. The result of the analysis is that there is a simple necessary and sufficient condition for $G$ to act freely and smoothly on some sphere:

Theorem 1.2 (Madsen-Thomas-Wall [58]). A finite group $G$ acts freely and smoothly on a sphere $S^{n}$ for some $n$ if and only if $G$ has periodic cohomology, and if, in addition, every subgroup of $G$ of order $2 p, p$ an odd prime, is cyclic.

However, it is not always easy to tell from knowledge of $G$ what is the minimal value of $n$. The necessity of the " $2 p$ condition" is due to Milnor [60], and follows from the following geometric result:

Theorem 1.3 (Milnor [60]). Let $T: S^{n} \rightarrow S^{n}$ be a map of period 2 without fixed points, and let $f: S^{n} \rightarrow S^{n}$ be a map of odd degree. Then there is a point $x \in S^{n}$ with $T f(x)=f T(x)$.

Proof of necessity of the Madsen-Thomas-Wall condition from Theorem 1.3. Suppose $G$ acts freely on a sphere and there is some subgroup $H$ of $G$ of order $2 p$ which is not cyclic. Then $H$ is dihedral. Let $T$ be the action of the generator of $H$ of order 2, and let $f$ be the action of the generator of $H$ of order $p$. Then by Theorem 1.3, $T f T^{-1} f^{-1}$ has a fixed point. Since $G$ acts freely, that means $T f T^{-1} f^{-1}=1$, so the two generators of $H$ commute with each other, a contradiction.

Another application is to the problem of when a non-compact manifold $M$ is homeomorphic to the interior of a compact manifold $W$ with boundary. Clearly this implies that $M$ should be homologically finite. Since any compact topological manifold $W$, even with boundary, has the homotopy type of a finite CW-complex, an additional necessary condition is that the Wall obstruction of $M$ should vanish. The highly influential thesis of Siebenmann [78] showed that in high dimensions, this condition and an obvious "tameness" condition are sufficient. 


\section{Flat Bundles and $K$-Theory}

Another connection between geometric topology (or more precisely, geometry and topology of manifolds) and algebraic $K$-theory comes from the study of flat vector bundles. Suppose $M$ is a smooth manifold and $E \rightarrow M$ is a smooth vector bundle over $M$. A connection on $E$ is a way of differentiating sections of $E$. More precisely, a connection is a map

$$
\nabla: \Gamma^{\infty}(E) \rightarrow \Gamma^{\infty}\left(E \otimes T^{*} M\right),
$$

where $\Gamma^{\infty}$ denotes "smooth sections," which we also think of as a bilinear pairing $\Gamma^{\infty}(E) \times \Gamma^{\infty}(T M) \rightarrow \Gamma^{\infty}(E),(s, X) \mapsto \nabla_{X}(s)$, satisfying the "Leibniz rule" $\nabla_{X}(f s)=X(f) \cdot s+f \nabla_{X}(s)$ for $f \in C^{\infty}(M)$. (See for example [29, pp. 56-60].) A connection is flat if it satisfies the analogue of the identity $d^{2}=0$ for the exterior derivative, or in other words if $\left[\nabla_{X}, \nabla_{Y}\right]=\nabla_{[X, Y]}$ for all vector fields $X$ and $Y$. This condition turns out to be equivalent [29, Cor. 3.22] to saying that there is a reduction of the structure group of the bundle from $G=G L(n, \mathbb{R})$ or $G L(n, \mathbb{C})$ to a discrete group. Now isomorphism classes of ordinary vector bundles are determined by their "transition functions," and are thus parameterized by the non-abelian sheaf cohomology group $H^{1}(M, \mathcal{G}), \mathcal{G}$ the sheaf of germs of $G$-valued functions on $M$. Equivalently, there are classified by homotopy classes of maps $M \rightarrow B G$. In the same way, isomorphism classes of flat vector bundles (where we keep track of the flat structure $\nabla$ ) are parameterized by non-abelian sheaf cohomology of the constant sheaf, $H^{1}\left(M, G^{\delta}\right)=\operatorname{Hom}\left(\pi_{1}(M), G^{\delta}\right)$ or by homotopy classes of maps $M \rightarrow B G^{\delta}$, where $G^{\delta}$ denotes $G$ with the discrete topology. Via the plus construction $B G L(n, \mathbb{C})^{\delta} \rightarrow B G L(\infty, \mathbb{C})^{\delta} \rightarrow B\left(G L(\infty, \mathbb{C})^{\delta}\right)^{+}$, we see that flat complex vector bundles give classes in $H^{0}(X ; \mathbb{K}(\mathbb{C}))$, the cohomology of $X$ with coefficients in the (algebraic, not topological) $K$-theory spectrum of $\mathbb{C}$. In particular, flat complex vector bundles over homology $n$-spheres can be viewed as representing classes in $H^{0}\left(S^{n} ; \mathbb{K}(\mathbb{C})\right)=\pi_{n}(\mathbb{K}(\mathbb{C}))=K_{n}(\mathbb{C})$, and it is easy to see that every class in $K_{n}(\mathbb{C})$ arises from some flat vector bundle over a homology $n$-sphere. In a similar vein, Hausmann and Vogel [40, Corollary 4.2] have shown that for any $\operatorname{ring} A$ and $n \geq 5, K_{n}(A)$ can be described as the "homology sphere bordism" of $B G L(A)$, i.e., as the group of equivalence classes of pairs $\left(\Sigma^{n}, f\right)$, where $\Sigma^{n}$ is a (based) oriented $n$-dimensional PL manifold which is an integral homology sphere ${ }^{2}, f: \Sigma^{n} \rightarrow B G L(A)$ (and sends basepoint to basepoint), and $\left(\Sigma_{1}^{n}, f_{1}\right) \simeq\left(\Sigma_{2}^{n}, f_{2}\right)$ if and only if there exists a compact manifold $W^{n+1}$ with $\partial M=\Sigma_{1} \amalg-\Sigma_{2}$, there exists a map $F: W \rightarrow B G L(A)$ extending $f_{1}$ and $f_{2}$ (sending a "base arc" joining the basepoints of the boundary components to the basepoint of $B G L(A)$ ), and the inclusions $\Sigma_{j} \hookrightarrow W$ are integral homology equivalences.

\footnotetext{
${ }^{2}$ We use PL manifolds rather than smooth ones to avoid complications coming
} from the finite group $\Theta_{n}$ of exotic $n$-spheres. 
We return again to the study of flat real or complex vector bundles. Suslin has shown [85] that for $k$ any infinite field (in particular for $k=\mathbb{R}$ or $\mathbb{C}$ ), the inclusion $G L(n, k)^{\delta} \hookrightarrow G L(\infty, k)^{\delta}$ induces an isomorphism on $H_{j}(\ldots ; \mathbb{Z})$ for $j \leq n$. Thus for studying characteristic classes of flat vector bundles on $n$-dimensional spaces, it's enough to look at flat vector bundles of rank $\leq n$. There are stability theorems saying that the map $B\left(G L(n, k)^{\delta}\right)^{+} \rightarrow$ $B\left(G L(\infty, k)^{\delta}\right)^{+}$is $(n / 2)$-connected, and it's plausible that this map is even $n$-connected. Hence for computing $K_{n}(\mathbb{R})$ or $K_{n}(\mathbb{C})$, it's enough to look at flat vector bundles of rank $\leq 2 n$, and it may even be that every class in $K_{n}(\mathbb{R})$ or $K_{n}(\mathbb{C})$ is represented by a flat vector bundle of rank $n$. But while the map $\pi_{n}\left(B\left(G L(n, \mathbb{R})^{\delta}\right)^{+}\right) \rightarrow K_{n}(\mathbb{R})$ may be surjective, it is known not to be injective; we will see why in a moment.

Various natural geometric questions about flat bundles can now be reduced (at least in part) to $K$-theory, and vice versa. (However, if one is interested in bundles not in the stable range, e.g., with rank equal to the dimension of the base space, then unstable $K$-theory is required.) We give only a few representative examples.

First we should say something about characteristic classes. A basic fact about flat vector bundles is that since the real (or rational) Chern or Pontrjagin classes of a vector bundle can be computed from the curvature of a connection using Chern-Weil theory, and since a flat connection has (by definition) curvature zero, these classes for a flat vector bundle necessarily vanish [29, 9.1 and 9.2]. Hence the Chern or Pontrjagin classes of a flat vector bundle are torsion. Since $K_{2 n}^{\text {top }}(\mathbb{C}) \cong \mathbb{Z}$ is determined by Chern classes, it follows that the natural map from algebraic to topological $K$-theory, $K_{2 n}(\mathbb{C}) \rightarrow K_{2 n}^{\text {top }}(\mathbb{C})$, coming from the obvious continuous map $G L(n, \mathbb{C})^{\delta} \rightarrow G L(n, \mathbb{C})$, vanishes for $n>0$. (However the map of spectra $\mathbb{K} \rightarrow \mathbb{K}^{\text {top }}$ induces isomorphisms on homotopy groups with finite coefficients by a famous theorem of Suslin [86], which is related to the fact that the Chern classes of flat bundles can carry non-trivial torsion information.)

One might guess on the basis of the above that all rational invariants of flat vector bundles have to vanish, but celebrated work of Milnor [61] shows that this is not the case for the Euler class of an oriented real vector bundle. More precisely, Milnor showed that if $M^{2}$ is a closed oriented surface of genus $g \geq 2$, so that the oriented rank-two real vector bundles $E$ over $M$ are classified by $\langle e(E),[M]\rangle \in \mathbb{Z}$, where $e(E)$ is the Euler class in $H^{2}(M, \mathbb{Z})$, then $E$ admits a flat connection if and only if $|\langle e(E),[M]\rangle|<g$. (See also $[29, \S 9$ and Corollary 9.18] for a nice exposition.) This theorem prompted a huge explosion of interest in characteristic classes of flat vector bundles. For example, Deligne and Sullivan [27] showed that every flat complex vector bundle over a finite CW-complex becomes trivial on some finite cover. Using some of the ideas of Milnor, Smillie [81] showed there are flat manifolds with non-zero Euler characteristic in all even dimensions greater than or equal to four. This in turn motivated a more complete study by Hausmann [39] of what manifolds can 
admit a flat structure, i.e., a flat connection on the tangent bundle. For example, he showed that (in dimension $\geq 5$ ) a stably parallelizable closed manifold $M^{2 m}$ is semi-s-cobordant to a manifold $M^{\prime}$ with a $\mathbb{Z}$-flat structure (coming from a map $\pi_{1}\left(M^{\prime}\right) \rightarrow B S L(2 m, \mathbb{Z})$ ) if and only if it is parallelizable. Here $M$ semi-s-cobordant to $M^{\prime}$ means that there is a compact manifold $W^{2 m+1}$ with boundary $\partial W=M \amalg M^{\prime}$ such that the inclusion $M \hookrightarrow W$ is a simple homotopy equivalence. (If the same is true for $M^{\prime} \hookrightarrow W$, then $M$ and $M^{\prime}$ are called $s$-cobordant, hence diffeomorphic if they have dimension $\geq 5$; see section 3 below.) In particular, every parallelizable closed manifold is homology-equivalent to a closed manifold with a $\mathbb{Z}$-flat structure. Hausmann's methods proved at the same time that the natural map $\pi_{n}\left(B\left(G L(n, \mathbb{R})^{\delta}\right)^{+}\right) \rightarrow K_{n}(\mathbb{R})$ cannot be injective for $n=2 m$ even, for the image of the Euler class $e$ under the restriction map $H^{n}(B S L(n, \mathbb{R}), \mathbb{Q}) \rightarrow H^{n}\left(B S L(n, \mathbb{R})^{\delta}, \mathbb{Q}\right)$ is non-zero on the image of the Hurewicz map $\pi_{n}\left(\left(B G L(n, \mathbb{R})^{\delta}\right)^{+}\right)=\pi_{n}\left(\left(B S L(n, \mathbb{R})^{\delta}\right)^{+}\right) \rightarrow$ $H_{n}\left(\left(B S L(n, \mathbb{R})^{\delta}\right)^{+}, \mathbb{Z}\right) \cong H_{n}\left(S L(n, \mathbb{R})^{\delta}, \mathbb{Z}\right)$, but does not lie in the image of the restriction map $H^{n}\left(B S L(\infty, \mathbb{R})^{\delta}, \mathbb{Q}\right) \rightarrow H^{n}\left(B S L(n, \mathbb{R})^{\delta}, \mathbb{Q}\right)$. Note that this now implies that the map $B\left(G L(n, \mathbb{R})^{\delta}\right)^{+} \rightarrow B\left(G L(\infty, \mathbb{R})^{\delta}\right)^{+}$cannot be $(n+1)$-connected.

The vanishing of rational characteristic classes of flat bundles makes it possible to define secondary characteristic classes, which can be used to detect some of the $K$-theory of fields. For simplicity we consider only complex vector bundles. From the long exact sequence

$$
\cdots \rightarrow H^{2 k-1}\left(X, \mathbb{C}^{\times}\right) \stackrel{\partial}{\rightarrow} H^{2 k}(X, \mathbb{Z}) \rightarrow H^{2 k}(X, \mathbb{C}) \rightarrow H^{2 k}\left(X, \mathbb{C}^{\times}\right) \stackrel{\partial}{\rightarrow} \cdots
$$

it follows that any integral torsion cohomology class in degree $2 k$ lifts to a class of degree $2 k-1$ with coefficients in $\mathbb{C}^{\times}$. A choice of such a lifting for the $k$-th Chern class of a flat rank- $n$ vector bundle $(E, \nabla)$ over $X$, defined using the flat connection $\nabla$, was (essentially) given by Chern and Simons [23], [24] and is called the Chern-Simons class. For example, a flat structure on a complex line bundle over $X$ is given simply by a homomorphism $\pi_{1}(X) \rightarrow \mathbb{C}^{\times}$, and thus defines a class in $H^{1}\left(X, \mathbb{C}^{\times}\right)$. In general, Chern and Simons consider the case, which one can always reduce to, where $X$ is a smooth manifold, and then they use the connection $\nabla$ to construct a closed differential form on the principal $G L(n)$-bundle associated to $E$, whose restriction to each fiber is integral. One can then view this form as defining a $\left(\mathbb{C} / \mathbb{Z} \cong \mathbb{C}^{\times}\right)$-valued class on the base. An alternative approach to the construction of the Chern-Simons classes for flat bundles may be found in [29, Exercise 3, pp. 163-164]. The approach there involves the space $F=G L(n, \mathbb{C}) / G L(k-1, \mathbb{C})$, which is $(2 k-2)$-connected and satisfies $H^{2 k-1}(F, \mathbb{Z}) \cong \mathbb{Z}$. For the reader's convenience, we fill in some of the missing details.

Proposition 2.1. The space $F=G L(n, \mathbb{C}) / G L(k-1, \mathbb{C})$ is $(2 k-2)$-connected and satisfies $H^{2 k-1}(F, \mathbb{Z}) \cong \mathbb{Z}$. 
Proof. There are deformation retractions from $G L(n, \mathbb{C})$ down to $U(n)$, and from $G L(k-1, \mathbb{C})$ down to $U(k-1)$. Since $U(k)$ acts transitively on the unit sphere $S^{2 k-1}$ in $\mathbb{C}^{k}$, with $U(k-1)$ the stabilizer of a point, $F$ has the homotopy type of $S^{2 k-1}$ when $k=n$, and then the result is obvious. If $n>k$, we have a fibration

$$
U(k) / U(k-1) \rightarrow U(n) / U(k-1) \rightarrow U(n) / U(k),
$$

and since $U(n) / U(k)$ is at least $2 k$-connected, the result follows.

The fact that $F$ is highly connected is then used as follows.

Proposition 2.2. Again let $F=G L(n, \mathbb{C}) / G L(k-1, \mathbb{C})$. There is a "filling" $\sigma$ of $F$ by singular simplices up through dimension $2 k-1$, or in other words a family of singular simplices

$$
\sigma\left(g_{1}, \cdots, g_{q}\right): \Delta^{q} \rightarrow F, \quad g_{1}, \cdots, g_{q} \in G L(n, \mathbb{C}), q \leq 2 k-1,
$$

which satisfy

$$
\sigma\left(g_{1}, \cdots, g_{q}\right) \circ \varepsilon^{i}= \begin{cases}g_{1} \cdot \sigma\left(g_{2}, \cdots, g_{q}\right), & i=0, \\ \sigma\left(g_{1}, \cdots, g_{i} g_{i+1}, \cdots, g_{q}\right), & 0<i<q, \\ \sigma\left(g_{1}, \cdots, g_{q-1}\right), & i=q .\end{cases}
$$

where the $\varepsilon^{i}$ are the face maps.

Proof. This is proved by induction on $q$. To start the induction, let $\sigma\left(\Delta^{0}\right)$ be the origin $o=e G L(k-1, \mathbb{C})$ in $F$. Assume $\sigma$ is defined for smaller values of $q$; then one can check that (2) defines $\sigma\left(g_{1}, \cdots, g_{q}\right)$ on the boundary of $\Delta^{q}$ in a consistent way. (For example, we need to check that the formulas for $\sigma\left(g_{1}, \cdots, g_{q}\right) \circ \varepsilon^{0}=g_{1} \cdot \sigma\left(g_{2}, \cdots, g_{q}\right)$ and for $\sigma\left(g_{1}, \cdots, g_{q}\right) \circ \varepsilon^{1}=\sigma\left(g_{1} g_{2}, \cdots, g_{q}\right)$ agree on the intersection of the 0 -th face and the 1-th face, which is a $(q-2)$ simplex. So we need to check that $g_{1} \cdot \sigma\left(g_{2}, \cdots, g_{q}\right) \circ \varepsilon^{0}=\sigma\left(g_{1} g_{2}, \cdots, g_{q}\right) \circ \varepsilon^{0}$; both are given by $g_{1} g_{2} \cdot \sigma\left(g_{3}, \cdots, g_{q}\right)$. The other verifications are similar.) Thus we just need to fill in. But for $q \leq 2 k-1, \pi_{q-1}(F)=0$, and thus any map $S^{q-1} \cong \partial \Delta^{q} \rightarrow F$ extends continuously to $\Delta^{q}$.

Proposition 2.3. There is a $G L(n, \mathbb{C})$-invariant closed $(2 k-1)$-form $\omega$ on $F$, representing the de Rham class of a generator of $H^{2 k-1}(F, \mathbb{Z})$.

Proof. Since $H^{2 k-1}(F, \mathbb{Z}) \cong \mathbb{Z}$ by Proposition 2.1, and in fact by the proof of that proposition there is a preferred generator (coming from the usual orientation of $S^{2 k-1}$ ), there is a canonical de Rham class representing this generator in $H^{2 k-1}(F, \mathbb{R})$. This de Rham class may be realized by a $U(n)$-invariant closed real form, since $U(n)$ is compact. (Just "average" any closed form in the de Rham class with respect to Haar measure on the compact group.) Then since $G L(n, \mathbb{C})$ is the complexification of $U(n)$ and acts transitively on $F$, we may complexify to a $G L(n, \mathbb{C})$-invariant complex closed form. 
Proposition 2.4. Define a group cochain $s$ on $G L(n, \mathbb{C})^{\delta}$ (with values in $\mathbb{C} / \mathbb{Z})$ by the formula

$$
s\left(g_{1}, \cdots, g_{2 k-1}\right)=\int_{\Delta^{2 k-1}} \sigma\left(g_{1}, \cdots, g_{2 k-1}\right)^{*}(\omega) \quad(\text { reduced } \bmod \mathbb{Z}) .
$$

Then $s$ is a cocycle and its cohomology class in $H^{2 k-1}\left(B G L(n, \mathbb{C})^{\delta}, \mathbb{C} / \mathbb{Z}\right)$ is a lifting of the $k$-th Chern class for flat bundles.

Proof. Let $G=G L(n, \mathbb{C})$. By definition,

$$
\begin{aligned}
\delta s\left(g_{1}, \cdots, g_{2 k}\right)= & s\left(g_{2}, \cdots, g_{2 k}\right) \\
& +\sum_{0<i<2 k}(-1)^{i} s\left(g_{1}, \cdots, g_{i} g_{i+1}, \cdots, g_{2 k}\right)+s\left(g_{1}, \cdots, g_{2 k-1}\right) \\
= & \int_{\Delta{ }^{2 k-1}}\left(\sigma\left(g_{2}, \cdots, g_{2 k}\right)^{*}(\omega)\right. \\
& +\sum_{0<i<2 k}(-1)^{i} \sigma\left(g_{1}, \cdots, g_{i} g_{i+1}, \cdots, g_{2 k}\right)^{*}(\omega) \\
& \left.+\sigma\left(g_{1}, \cdots, g_{2 k-1}\right)^{*}(\omega)\right) \\
= & \int_{C\left(g_{1}, \cdots, g_{2 k}\right)} \omega,
\end{aligned}
$$

where $C\left(g_{1}, \cdots, g_{2 k}\right)$ is the singular chain

$g_{1} \cdot \sigma\left(g_{2}, \cdots, g_{2 k}\right)+\sum_{0<i<2 k}(-1)^{i} \sigma\left(g_{1}, \cdots, g_{i} g_{i+1}, \cdots, g_{2 k}\right)+\sigma\left(g_{1}, \cdots, g_{2 k-1}\right)$.

(Note that we've used $G$-invariance of $\omega$ to replace $\sigma\left(g_{2}, \cdots, g_{2 k}\right)$ by $g_{1}$. $\sigma\left(g_{2}, \cdots, g_{2 k}\right)$ here.) By the defining property (2) of $\sigma, C\left(g_{1}, \cdots, g_{2 k}\right)$ is a singular cycle. But $\omega$ represents an integral de Rham class, so its integral over $C\left(g_{1}, \cdots, g_{2 k}\right)$ vanishes in $\mathbb{C} / \mathbb{Z}$. Thus $s$ is a group cocycle.

It remains to show that $\partial[s]=c_{k}$ in the sequence (1). But by the calculation in (3), $\partial[s]$ is represented by the group cocycle whose value on $\left(g_{1}, \cdots, g_{2 k}\right)$ is given by $\int_{C\left(g_{1}, \cdots, g_{2 k}\right)} \omega, C\left(g_{1}, \cdots, g_{2 k}\right)$ as above. We can see that this is the primary obstruction to triviality of the universal bundle over $B G^{\delta}$ with fiber $F$ (associated to the universal principal $G$-bundle over $B G^{\delta}$ ). Indeed, it was the homotopy group $\pi_{2 k-1}(F)$ which in the proof of Proposition 2.2 gave the obstruction to extending the filling $\sigma$ to dimension $2 k$, and had we been able to do this, $C\left(g_{1}, \cdots, g_{2 k}\right)$ would be the boundary of $\sigma\left(g_{1}, \cdots, g_{2 k}\right)$ and thus $\int_{C\left(g_{1}, \cdots, g_{2 k}\right)} \omega$ would have vanished. The definition of Chern classes by obstruction theory then gives the result. 


\section{Whitehead and Reidemeister Torsion}

One of the early sources for the development of $K$-theory is the geometric invariant known as Whitehead torsion, for which convenient textbook treatments are [25] and [76]. However, the best condensed reference is still probably Milnor's classic survey article, [63]. Another good exposition is in [64]. In its essence, the idea of Whitehead torsion is to measure the extent to which a given homotopy equivalence, say between finite polyhedra, is of the "trivial" sort. Here "trivial" homotopy equivalences are generated by three basic kinds of operations: simplicial homeomorphisms (possibly after subdivision of some simplices) and elementary expansions and collapses. Expansions and their duals, collapses, are best illustrated by a picture (Fig. 1).

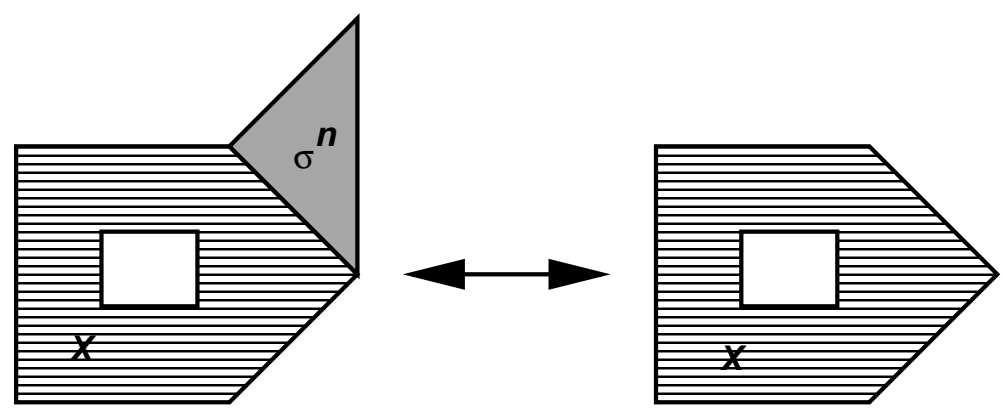

Fig. 1. An elementary expansion (or collapse, depending on whether one reads the picture from right to left or left to right)

In other words, we say $X^{\prime}$ collapses to $X$ if $X^{\prime}=X \cup \sigma^{n}$, where $\sigma^{n}$ is an $n$-simplex attached to $X$ along one of its codimension-one faces, and then clearly we can "squash" $X^{\prime}$ down to $X$, and this gives a homotopy equivalence from $X^{\prime}$ to $X$. A homotopy equivalence between finite polyhedra is called simple if it can be constructed out of a chain of simplicial homeomorphisms (after subdivision) and elementary collapses and expansions. There is a similar notion for finite $\mathrm{CW}$-complexes as well: in the CW-context, $X^{\prime}$ collapses to $X$ if $X^{\prime}$ is obtained from $X$ by attaching first an $(n-1)$-cell with a nullhomotopic attaching map $S^{n-2} \rightarrow X$, and then an $n$-cell bounded in the obvious way by this $(n-1)$-cell, the same way $D^{n}$ is bounded by $S^{n-1}$. It is easy to see that a polyhedral collapse is a special case of a cellular collapse, since attaching $\sigma^{n}$ to $X$ as in Fig. 1 is the same as first attaching the boundary of $\sigma^{n}$ and then filling in with an $n$-cell. Any homotopy equivalence $h: X \rightarrow X^{\prime}$ of (connected) finite polyhedra or finite $\mathrm{CW}$-complexes has an invariant $\tau(h) \in \mathrm{Wh}\left(\pi_{1}(X)\right)$, where $\mathrm{Wh}(\pi)$ is a certain quotient of $K_{1}(\mathbb{Z} \pi)$, and this invariant is trivial exactly when $h$ is simple. We will content ourselves with describing this invariant in the simplest case. If $h$ is an inclusion map and $\left(X^{\prime}, X\right)$ is a finite relative $\mathrm{CW}$-complex, with all relative cells of dimensions 
$n-1$ and $n$ (so that $X^{\prime}$ is obtained from $X$ by attaching first $(n-1)$-cells and then $n$-cells), then since $h$ is a homotopy equivalence, the relative cellular chain complex of the universal covers, $C_{*}\left(\widetilde{X}^{\prime}, \widetilde{X}\right)$, reduces simply to an isomorphism $\partial: C_{n} \rightarrow C_{n-1}$ of finitely generated free $\mathbb{Z} \pi_{1}(X)$-modules. We have obvious bases for the chain modules $C_{n}$ and $C_{n-1}$ which only depend on a choice of orientation for each relative cell of $\left(X^{\prime}, X\right)$ and a choice of an inverse image for this cell in $\widetilde{X}^{\prime}$. Since the cellular boundary map $\partial$ is an isomorphism, one can think of $\partial$ as defining an invertible matrix with entries in $\mathbb{Z} \pi_{1}(X)$. Now of course the matrix depends on the choice of bases for the free $\mathbb{Z} \pi_{1}(X)$ modules involved, but the ambiguity in the choice only affects the $K_{1}$-class of the matrix by at most a sign (coming from the choices of orientations) and an element of $\pi_{1}(X)$ (coming from the choices of inverse images in $\tilde{X}^{\prime}$ for the cells of $\left.\left(X^{\prime}, X\right)\right)$. Thus if we define $\mathrm{Wh}\left(\pi_{1}(X)\right)$ to be the quotient of $K_{1}\left(\mathbb{Z} \pi_{1}(X)\right)$ by the subgroup generated by the canonical images of $\mathbb{Z}^{\times} \cong\{ \pm 1\}$ and of $\pi_{1}(X)$, we obtain an invariant in this group independent of all choices. ${ }^{3}$ One can show that this invariant vanishes if and only if the homotopy equivalence is simple. The "if" direction is rather straightforward from the definitions. The "only if" definition requires showing that that an elementary matrix corresponds geometrically to a collapse. (This requires "unhooking" one of the cells involved.)

It would appear that the construction of Whitehead torsion is highly dependent on a choice of simplicial or cellular structures for the spaces involved, but a deep and surprising theorem of Chapman says that this dependence is illusory.

Theorem 3.1 (Chapman [19]). If $X$ and $X^{\prime}$ are connected finite polyhedra and $h: X \rightarrow X^{\prime}$ is a (simplicial) homotopy equivalence, then $\tau(h)$ is a topological invariant. In other words, if we can fit $h$ into a commutative diagram

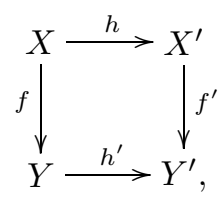

where $Y$ and $Y^{\prime}$ are also finite polyhedra, $h^{\prime}$ is a simplicial homotopy equivalence, and $f$ and $f^{\prime}$ are homeomorphisms, then $\tau(h)=\tau\left(h^{\prime}\right)$.

This suggests that Whitehead torsion has some deeper significance, and in fact it plays a basic role in the classification of manifolds, for the following reason. If $M$ and $M^{\prime}$ are compact connected $n$-manifolds (smooth, let's say), an $(n+1)$-manifold with boundary $W$ is called a cobordism between $M$ and $M^{\prime}$ if $\partial W=M \amalg M^{\prime}$. (If $W, M$, and $M^{\prime}$ are oriented, then $W$ should

\footnotetext{
${ }^{3}$ Strictly speaking, $\tau\left(X^{\prime}, X\right)$ is this class multiplied by a sign depending on the parity of $n$; that's because when there are relative cells of many dimensions, what we want is a kind of multiplicative analogue of the Euler characteristic.
} 
induce the opposite of the given orientation on $M^{\prime}$, so that $M \times[0,1]$ is an allowable cobordism from $M$ to itself.) We call $W$ an $h$-cobordism if the inclusions $M \hookrightarrow W$ and $M^{\prime} \hookrightarrow W$ are homotopy equivalences, in which cases the torsions $\tau(W, M)$ and $\tau\left(W, M^{\prime}\right)$ are defined. We call $W$ an $s$-cobordism if the inclusions $M \hookrightarrow W$ and $M^{\prime} \hookrightarrow W$ are simple homotopy equivalences, i.e., the torsions $\tau(W, M)$ and $\tau\left(W, M^{\prime}\right)$ both vanish. An $h$-cobordism is called trivial if it is diffeomorphic to $M \times[0,1]$. When this is the case, note that $M^{\prime}$ is automatically diffeomorphic to $M$, and $\tau(W, M)$ and $\tau\left(W, M^{\prime}\right)$ are both trivial. Smale's famous $h$-cobordism theorem [62] asserts that every simply connected $h$-cobordism is trivial if $n \geq 5$. However, this cannot possibly be true in the non-simply connected case because of the Whitehead torsion obstruction, and the substitute is the $s$-cobordism theorem.

Theorem 3.2 (s-cobordism theorem [52]). Suppose $W^{n+1}$ is an $h$-cobordism between connected smooth manifolds $M$ and $M^{\prime}$, and suppose $n \geq 5$. If $\tau(W, M)=0$, then $W$ is trivial. Moreover, if $n \geq 5$, then every element of $\mathrm{Wh}\left(\pi_{1}(M)\right)$ can be realized by an $h$-cobordism from $M$ to some homotopyequivalent manifold $M^{\prime}$.

The same statement holds in the PL category, for which a suitable reference is [76], and even (thanks to the work of Kirby-Siebenmann [54]) in the topological category.

The importance of Whitehead torsion for geometric topology makes it important to understand the Whitehead group $\mathrm{Wh}(\pi)$ for various classes of groups $\pi$. It is not too hard to prove that $\mathrm{Wh}(\pi)=0$ for $\pi$ of order $\leq 4$ and that $\operatorname{Wh}(\pi)$ is infinite cyclic for $\pi$ of order 5 . More generally, the most basic fact about the Whitehead group for finite groups is:

Theorem 3.3 (Bass - see [66], Theorems 2.5 and 2.6). Suppose $\pi$ is a finite group. Then $\mathrm{Wh}(\pi)$ is finitely generated, and $\operatorname{rk}(\mathrm{Wh}(\pi))$ is the difference between the number of irreducible representations of $\pi$ over $\mathbb{R}$ and the number of irreducible representations of $\pi$ over $\mathbb{Q}$.

Just as an example, if $\pi$ is of order $p$, an odd prime, $\pi$ has $(p-1) / 2$ inequivalent two-dimensional irreducible representations over $\mathbb{R}$, but one $(p-$ 1)-dimensional irreducible representation over $\mathbb{Q}$ (since $\mathbb{Q} \pi \cong \mathbb{Q} \times \mathbb{Q}(\zeta), \zeta$ a primitive $p$-th root of unity, and $[\mathbb{Q}(\zeta): \mathbb{Q}]=p-1)$, so $\operatorname{rk}(\mathrm{Wh}(\pi))=$ $\frac{p-1}{2}+1-2=(p-3) / 2$.

However, computing the exact structure of $\mathrm{Wh}(\pi)$ for finite groups $\pi$ is difficult, though in principle understood. The best survey on this is the book [66] by Oliver.

For infinite groups $\pi$, there is a widespread belief that $\mathrm{Wh}(\pi)$ should be attributable to the torsion in $\pi$. (For an exact formulation of a conjecture to this effect, see the chapter by Lück and Reich.) But still open is the most fundamental version of this conjecture:

Conjecture 3.4. The Whitehead group $\mathrm{Wh}(\pi)$ vanishes for any torsion-free group $\pi$. 
There are many situations in geometric topology where Whitehead torsion is not well defined, but one can still define a torsion-like invariant called Reidemeister torsion. For example, if $X$ is a finite connected CW-complex with fundamental group $\pi$, it may be that the reduced cellular chain complex $\bar{C}_{*}(\widetilde{X})$ is not acyclic (i.e., $H_{j}(X, \mathbb{Z} \pi) \neq 0$ for some $j>0$ ), so that $\tau(X, *)$ is undefined, and yet $C_{*}(X, V)$ may be acyclic for some local coefficient system $V$. In this case, we can define the Reidemeister torsion of $X$ with coefficients in $V$. Roughly speaking, the difference between Whitehead and Reidemeister torsion is this. An $n \times n$ matrix $a$ with entries in $\mathbb{Z} \pi$ defines a class in $\mathrm{Wh}(\pi)$ if the matrix is invertible. However, it may be that the matrix is not invertible, but its image under some representation of $\pi$ is invertible. For example, suppose one has an orthogonal or unitary representation $\pi \rightarrow O(m)$ or $\pi \rightarrow U(m)$. Then this induces a ring homomorphism $\mathbb{Z} \pi \rightarrow M_{m}(\mathbb{R})$ or $M_{m}(\mathbb{C})$, under which the group of units $\mathbb{Z}^{\times} \times \pi$ maps to matrices with determinant of absolute value 1. So the absolute value of the determinant $|\operatorname{det}(a)| \in \mathbb{R}_{+}^{\times}$is unchanged if we change $a$ by an element of the image of $\mathbb{Z}^{\times} \times \pi \subseteq G L(1, \mathbb{Z} \pi) \hookrightarrow G L(n, \mathbb{Z} \pi)$. The simplest geometric example is the case of $X=S^{1}$ and the representation of $\pi_{1}\left(S^{1}\right) \cong \mathbb{Z}$ sending the generator to $e^{i \theta}, 0<\theta<2 \pi$. The cellular chain complex of $S^{1}$ with coefficients in the associated local system is $\mathbb{C} \stackrel{e^{i \theta}-1}{\longrightarrow} \mathbb{C}$, so the complex is acyclic (under the assumption $0<\theta<2 \pi$ ) and the torsion is $\left|e^{i \theta}-1\right|=2|\sin (\theta / 2)|$.

There are two important classical examples of Reidemeister torsion. If $X$ is the complement of a knot in $S^{3}$ and one takes the representation $\pi_{1}(X) \rightarrow \mathbb{C}^{\times}$ sending a generator of $H_{1}(X)=\pi_{1}(X)_{\mathrm{ab}} \cong \mathbb{Z}$ to a transcendental number $t$, then the Reidemeister torsion becomes essentially (except for a trivial factor) the Alexander polynomial $\Delta(t)$ of the knot [63, Example 2, p. 387]. The second important case is where $X$ is a lens space, the quotient of $S^{2 n-1}$ by a free linear action of a cyclic group $\pi=\mathbb{Z} / m$ on $\mathbb{C}^{n}$. In this case, the Reidemeister torsion is the essential invariant for classifying lens spaces with fixed dimension and fundamental group up to homeomorphism. More precisely (see [63, §12] for details), the lens spaces with fundamental group $\pi$ and dimension $2 n-1$ are classified by $n$ elements $r_{1}, \cdots, r_{n} \in(\mathbb{Z} / m)^{\times}$, modulo a certain equivalence relation, and the Reidemeister torsion (for the representation of $\pi$ sending the generator to a primitive $m$-th root of unity $t$ ) turns out to be

$$
\prod_{j=1}^{n}\left(t^{r_{j}}-1\right)
$$

modulo multiplication by factors of $\pm t^{k}$. The torsion is of course an invariant of the simple homotopy type, and by Chapman's Theorem (Theorem 3.1), even a homeomorphism invariant. From this one can prove that two lens spaces are homeomorphic if and only if they are isometric, which is certainly not obvious. (On the other hand, there are plenty of examples of lens spaces which are homotopy equivalent but not simple homotopy equivalent, and also 
plenty of examples of lens spaces with the same dimension and fundamental group which are not even homotopy equivalent.)

One of the most remarkable things about Reidemeister torsion is its relation to a global analytic invariant in Riemannian geometry, the analytic torsion of Ray and Singer [73]. Ray and Singer defined the analytic torsion by reformulating the definition of the Reidemeister torsion in terms of the "combinatorial Laplacian," then replacing this operator in the definition by the Laplace-Beltrami operator of Riemannian geometry. They conjectured that the resulting invariant, given in terms of the spectrum of the Laplacian on differential forms, coincides with the Reidemeister torsion, and this conjecture was eventually proven by Cheeger [22] and Müller [65], working independently.

Various generalizations of the Cheeger-Müller theorem, for example, replacing ordinary determinants by the Kadison-Fuglede determinant ${ }^{4}$ on a finite von Neumann algebra (e.g., [12]), or allowing manifolds with boundary or non-compact manifolds, are a major topic of current research.

\section{Controlled $K$-Theory and Connections with Negative $K$-Theory}

One of the most interesting areas where algebraic $K$-theory and geometric topology come together is in the subject of controlled $K$-theory. In this theory, one studies not just projective modules over a ring and morphisms between them, but also the effect of imposing conditions on the "placement" or "support" of the modules or morphisms.

Probably the simplest example of controlled $K$-theory is an elegant description of negative $K$-theory by Pedersen [69], which led to a description by Pedersen and Weibel [68], [67] of the homology theory attached to the (nonconnective) $K$-theory spectrum $\mathbb{K}(R)$ of a ring $R$. These examples lead to what is often called $K$-theory with bounded control. Say one is given a ring $R$ and a (non-empty) metric space $(X, d)$. One considers the category $\mathcal{C}_{X}(R)$ of "locally finitely generated" configurations of projective modules over $X$, i.e., maps $x \mapsto P_{x}$ from $X$ to finitely generated projective $R$-modules, such that $\bigoplus_{x \in B} P_{x}$ is finitely generated for each set $B \subseteq X$ of finite diameter. Morphisms are $R$-module endomorphisms of $\bigoplus_{x \in X} P_{x}$ whose component $P_{x} \rightarrow P_{y}$ vanishes once $d(x, y)$ is sufficiently large. Applying the usual $K$ theoretic constructions gives a $K$-theory spectrum $\mathbb{K}(R ; X)$ and thus groups $K_{i}(R ; X)=\pi_{i}(\mathbb{K}(R ; X))$. Here only the "large scale" geometry of $X$ is relevant. For example, if $X$ has finite diameter, $\mathbb{K}(R ; X) \simeq \mathbb{K}(R ; \mathrm{pt})=\mathbb{K}(R)$, and similarly $\mathbb{K}\left(R ; \mathbb{R}^{n}\right) \simeq \mathbb{K}\left(R ; \mathbb{Z}^{n}\right)$ (if $\mathbb{R}^{n}$ and $\mathbb{Z}^{n}$ are given the usual metrics). In this language, the main theorem of [69] asserts that $\mathbb{K}\left(R ; \mathbb{Z}^{n}\right)$ is the usual nonconnective $n$-fold delooping of $\mathbb{K}(R)$, and thus $K_{0}\left(R ; \mathbb{Z}^{n}\right) \cong K_{-n}(R)$. Then the papers [68] and [67] go on to show that if $\mathcal{O}(Y)$ is the infinite open cone

\footnotetext{
${ }^{4} \mathrm{On}$ a $\mathrm{II}_{1}$ factor $A$, this "determinant" gives an isomorphism $K_{1}(A) \rightarrow \mathbb{R}_{+}^{\times}[57]$.
} 
on a compact space $Y$, with the usual metric (so that if $Y$ is embedded in $S^{n-1} \subset \mathbb{R}^{n}, \mathcal{O}(Y)$ is an $\mathbb{R}_{+}^{\times}$-invariant subset of $\mathbb{R}^{n}$, from which it inherits the induced metric), then $K_{i}(R ; \mathcal{O}(Y)) \cong \widetilde{H}_{i-1}(Y ; \mathbb{K}(R))$.

The boundedly controlled $K$-theory $\mathbb{K}(R ; X)$ appears in many geometric applications, both directly and implicitly. Examples include the thin $h$ cobordism theorem of Quinn [70, Theorem 2.7] (this predated the above formulation of the theory, but involves some of the same ideas), the bounded $s$-cobordism theorem of Ferry and Pedersen [34, Theorem 2.17], and the work of Gunnar Carlsson [16] on the $K$-theoretic version of the Novikov conjecture. (See also Carlsson's chapter in this volume for more details.)

For applications to geometric topology, sometimes $K$-theory with $e p$ silon control is more relevant. The best motivation for this subject is the Chapman-Ferry Theorem ([32], [21]), which asserts that a homotopy equivalence $h: M^{\prime} \rightarrow M$ between closed manifolds $M$ and $M^{\prime}$ of dimension $n \geq 5$ is homotopic to a homeomorphism once it is "sufficiently controlled." To explain what this means, recall that the definition of a homotopy equivalence means that there is a map $h^{\prime}: M \rightarrow M^{\prime}$ and there are homotopies $H_{1}: h \circ h^{\prime} \simeq \mathrm{id}_{M}$, $H_{2}: h^{\prime} \circ h \simeq \operatorname{id}_{M^{\prime}}$. For $h$ to be "sufficiently controlled" means that if we fix a metric $d$ on $M, d\left(H_{1}(x, t), x\right) \leq \varepsilon$ and $d\left(h \circ H_{2}(y, t), h(y)\right) \leq \varepsilon$ for all $x \in M$, $y \in M^{\prime}$, and all $t \in[0,1]$. The theorem asserts that given $M$ and $d$, there is a $\varepsilon>0$ such that all $\varepsilon$-controlled homotopy equivalences $h: M^{\prime} \rightarrow M$ are homotopic (even $\varepsilon$-homotopic) to homeomorphisms. While neither the statement not the proof of the Chapman-Ferry Theorem involves $K$-theory directly, one can see that there has to be a connection. In fact, for the theorem to be true, it is clearly necessary (because of Theorem 3.1) for $\tau(h)=0 \in \mathrm{Wh}\left(\pi_{1}(M)\right)$ once $h$ is sufficiently controlled, which is not immediately obvious.

A treatment of $\varepsilon$-controlled Whitehead torsion and an associated controlled $s$-cobordism theorem $[20, \S 14]$ many be found in [20]. Chapman also states and proves $[20, \S \S 6-8]$ an $\varepsilon$-controlled version of the Wall finiteness obstruction (Theorem 1.1). This concerns the situation where one has a space $X$ with a reference map $p: X \rightarrow B, B$ a metric space. We say $X$ is $\varepsilon$ dominated by a space $Y$ if there are maps $f: X \rightarrow Y$ and $g: Y \rightarrow X$ such that $g \circ f \simeq_{\varepsilon} 1_{X}$, where the symbol $\simeq_{\varepsilon}$ denotes "is $\varepsilon$-homotopic to," i.e., there is a homotopy whose composition with $p$ doesn't move points more than a distance $\varepsilon$. Chapman answers the question of when an $\varepsilon$-finitely dominated space is $\varepsilon$-homotopy equivalent to a finite polyhedron.

One can formulate many similar theorems that involve controlled versions of Whitehead torsion or similar $K$-theoretic obstructions. Examples are the thin $h$-cobordism theorem of Quinn ([70, Theorem 2.7] and [71, Theorem 2.1.1]). 


\section{Equivariant and Stratified Situations}

So far, we have mostly discussed the topology of smooth, topological, or PL manifolds just by themselves. But $K$-theory also comes into play in the study of actions of groups (let's say finite groups for simplicity) on such manifolds, or in the study of stratified spaces such as complex algebraic or analytic varieties. (Such a variety has a dense open subset which is smooth; the complement of this smooth set, the singular set, is of smaller dimension and itself contains a dense smooth set, etc.) The connection between these two topics may be seen in the fact that if a finite group $G$ acts (smoothly, say) on a manifold $M$, then there is a dense open subset consisting of "principal orbits" (where the stabilizers are as small as possible), and once again the complement of this set is of smaller dimension and consists of more "singular" orbits.

The simplest example of a singular space is the one-point compactification $X=M_{+}$of a non-compact manifold $M$, or equivalently, a compact space with exactly one singular point. Detailed study of this example can tell us much about the general case. Just as an example, a natural question is how to formulate the $s$-cobordism theorem for such spaces. This problem is clearly equivalent to that of formulating a (proper) $s$-cobordism theorem for noncompact manifolds, which was done by Siebenmann in [79]:

Theorem 5.1 (proper s-cobordism theorem [79]). Suppose $W^{n+1}$ is a proper $h$-cobordism between connected smooth non-compact manifolds $M$ and $M^{\prime}$, and suppose $n \geq 5$. (In other words, $\partial W=M \amalg M^{\prime}$, and the inclusions $M \hookrightarrow W$ and $M^{\prime} \hookrightarrow W$ are proper homotopy equivalences. Then a Whitehead torsion invariant $\tau(W, M)$ is defined in a group $\mathrm{Wh}^{p}(M)$, and if $\tau(W, M)=0$, then $W$ is isomorphic (in the appropriate category) to $M \times[0,1]$. Moreover, if $n \geq 5$, then every element of $\mathrm{Wh}^{p}(M)$ is realized by an $h$-cobordism. Assuming for simplicity that $M$ has one end $\mathcal{E}$ and that $\mathcal{E}$ is tame, i.e., that for sufficiently large compact $C \subset M, M \backslash C$ is connected and its fundamental group $\pi_{1}(\mathcal{E})$ is independent of $C$, the group $\mathrm{Wh}^{p}(M)$ fits into an exact sequence

$$
\mathrm{Wh}\left(\pi_{1}(\mathcal{E})\right) \rightarrow \mathrm{Wh}\left(\pi_{1}(M)\right) \rightarrow \mathrm{Wh}^{p}(M) \rightarrow \widetilde{K}_{0}\left(\mathbb{Z} \pi_{1}(\mathcal{E})\right) \rightarrow \widetilde{K}_{0}\left(\mathbb{Z} \pi_{1}(M)\right) .
$$

A direct algebraic description of the obstruction group $\mathrm{Wh}^{p}(M)$ is given in [31].

A non-obvious corollary of this theorem is that simple homotopy type has a geometrical meaning: two finite-dimensional $\mathrm{CW}$-complexes have the same simple homotopy type if and only if they have piecewise linearly homeomorphic (closed) regular neighborhoods in some Euclidean spaces. (For finite CW-complexes this result is classical and is discussed in [103, pp. 22-23].) One direction is clear: if $X$ and $X^{\prime}$ are finite-dimensional CW-complexes with piecewise linearly homeomorphic (closed) regular neighborhoods, then since a PL homeomorphism is simple, we obtain a simple homotopy equivalence from $X$ to $X^{\prime}$ (via the intermediary of the regular neighborhoods). To prove 
the other direction, observe that a simple homotopy equivalence $X \approx X^{\prime}$ can without loss of generality be taken to be the inclusion of one end of a mapping cylinder. Taking a regular neighborhood in a sufficiently large Euclidean space, one can convert this mapping cylinder into a proper $h$-cobordism, where the two ends of the cobordism are regular neighborhoods of $X$ and $X^{\prime}$. Then simplicity of $X \approx X^{\prime}$ says by Theorem 5.1 that the $h$-cobordism is a product, and the result follows.

Next, we discuss some applications of algebraic $K$-theory to the study of actions of finite groups on complexes or manifolds. Some of this could be (and has been) generalized to actions of more general compact Lie groups or to proper actions of infinite discrete groups, but even the case of finite groups is too complicated to treat in detail here.

An easy place to begin is with the Wall finiteness obstruction. Let $G$ be a finite group and let $X$ be a $G$-CW-complex. The notions of finite domination and finiteness make sense in the equivariant world (we replace homotopies by $G$-homotopies, homotopy equivalences by $G$-homotopy equivalences). So it is natural to ask, assuming $X$ is $G$-dominated by a finite $G$-CW-complex, whether $X$ is $G$-homotopy equivalent to a finite $G$-CW-complex. One case we have effectively already done - if $X$ is connected and simply connected and the action of $G$ on $X$ is free, so $Y=X / G$ has fundamental group $G$, then this reduces to the question of whether $Y$ is finitely dominated, which is the case if and only of the usual Wall obstruction in $\widetilde{K}_{0}(\mathbb{Z} G)$ vanishes. The more general situation was first treated by Baglivo [6], who studied the case where $X$ is connected in the equivariant sense, i.e., where $X^{H}$ is connected and nonempty for every subgroup $H \subseteq G$. More general cases were treated by Lück [56] and others - see [3] for a survey of the many approaches.

The equivariant Wall obstruction appears in a number of problems about group actions, in combination with the Swan homomorphism

$$
\sigma:(\mathbb{Z} /|G|)^{\times} \rightarrow \widetilde{K}_{0}(\mathbb{Z} G),
$$

the boundary map $\sigma$ in the Mayer-Vietoris sequence in $K$-theory

$$
\cdots \rightarrow K_{1}(\mathbb{Z}) \oplus K_{1}(\mathbb{Z} G /(n)) \rightarrow K_{1}(\mathbb{Z} /|G|) \stackrel{\sigma}{\rightarrow} \widetilde{K}_{0}(\mathbb{Z} G) \rightarrow \cdots
$$

associated to the pull-back square

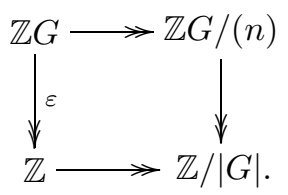

Here $\varepsilon: \mathbb{Z} G \rightarrow \mathbb{Z}$ is the augmentation map (sending each element of $G$ to 1 ) and $n=\sum_{g \in G} g$ is the "norm element" of $\mathbb{Z} G$. The relevance of the map $\sigma$ in this context was first noticed in [5]. 
Let $G$ be a finite group and let $X$ be a $G$-CW-complex. Then $X$ is called Smith acyclic if, for each subgroup $H$ of $G$ of prime power order $p^{r}, r \geq 1$, $\widetilde{H}_{*}\left(X^{H}, \mathbb{F}_{p}\right)=0$. A famous result of P. A. Smith [82] says that the singular set (the set of points with non-trivial stabilizer) of an action of $G$ on a finitedimensional contractible space is Smith acyclic, and it is natural to ask about the converse.

Theorem 5.2 ([5], Proposition 0.4). Let $G$ a finite group, and let $X$ be a Smith acyclic finite $G$-CW-complex for which every point has a non-trivial stabilizer. Then $X$ is the singular set for an action of $G$ on a contractible finite $G$-CW-complex if and only if

$$
\sum_{i}(-1)^{i} \sigma\left(\widetilde{H}_{i}(X, \mathbb{Z} /|G|)\right)=0
$$

in $\widetilde{K}_{0}(\mathbb{Z} G)$. (The Smith acyclicity of $X$ implies that each $\widetilde{H}_{i}(X, \mathbb{Z} /|G|)$ is of order prime to $|G|$, so that we can think of it as representing an element of $(\mathbb{Z} /|G|)^{\times}$, and thus (4) makes sense.)

The "only if" direction of this theorem follows from making precise the equivariant Wall obstruction. If "if" direction is proved by a direct inductive construction, where we add equivariant cells of type $e^{i} \times G$ to $X$, analogous to the proof of Theorem 1.1.

Theorem 5.2 paved the way for the study of many problems about extension of group actions and "homology propagation." The latter has to do with showing that, roughly speaking, if two manifolds have similar homology, then they carry similar group actions. Results of this type may be found in [5], [102], [14], and [15], just to cite a few sources.

Still another application of the equivariant Wall finiteness obstruction, but one requiring controlled topology also, may be found in a dramatic theorem of Steinberger and West [84]: a locally linear action of a finite group $G$ on a manifold $M$, assuming all components of the fixed point sets of all subgroups have dimension $\geq 6$ and none has codimension 1 or 2 in another, can be given an equivariant handle structure if and only if, for each $\varepsilon>0, M$ is equivariantly $\varepsilon$-homotopy equivalent to a finite $G$-CW-complex.

The equivariant Whitehead group $\mathrm{Wh}_{G}(X)$ and its basic properties were defined by Illman [50]. Anderson [1], Hauschild [38], and Illman later [51] showed that the equivariant Whitehead group $\mathrm{Wh}_{G}(X)$ can be expressed as a direct sum of ordinary Whitehead groups $\mathrm{Wh}\left((W H)_{\alpha}^{*}\right)$. The sum is over equivalence classes of connected components $X_{\alpha}^{H}$ of fixed sets $X^{H}$, where $H$ runs over the subgroups of $G$. The group $(W H)_{\alpha}$ is defined as $(W H)_{\alpha}=\{w \in$ $\left.W H: w \cdot X_{\alpha}^{H}=X_{\alpha}^{H}\right\}$, where $W H=N_{G}(H) / H$. Finally, the group $(W H)_{\alpha}^{*}$ is an extension of $(W H)_{\alpha}$ by $\pi_{1}\left(X_{\alpha}^{H}\right)$. As expected, the equivariant Whitehead group appears in the equivariant $s$-cobordism theorem in [83] and in [4].

Finally, we return to the case of more general stratified spaces. This case gets to be quite complicated, and the best place for the novice to begin is 
first with the survey article [42] and then with Weinberger's book [103]. As explained in [42, §1], many definitions and categories of stratified sets have been proposed. In all cases, we want to consider locally finite partitions $\Sigma$ of a locally compact, separable metric space $X$ into pairwise disjoint, locally closed subsets $X_{i}$, called the (pure) strata, each of which is a topological manifold, with $\operatorname{cl} X_{i} \cap X_{j} \neq \emptyset$ if and only if $X_{j} \subseteq \operatorname{cl} X_{i}$. The index set is then partially ordered by $j \leq i$ if and only if $X_{j} \subseteq \operatorname{cl} X_{i}$. The closed sets $\operatorname{cl} X_{i}$ are often called the closed strata. The differences between the various categories of stratified spaces have to do with "gluing" conditions on how the strata are joined. Essentially all of the definitions apply to "good" stratified spaces, like projective algebraic varieties over $\mathbb{C}$, but they do not necessarily apply to orbit spaces of finite groups acting locally linearly on topological manifolds, where one needs a weak form of the definition.

For many purposes, the best theory of stratified spaces to use is that of Browder and Quinn [11] — see also [103, §§6-10]. In this theory one keeps track of mapping cylinder neighborhoods. In other words, if $X_{i}$ is a stratum and

$$
\Sigma X_{i}=_{\text {def }}\left(\operatorname{cl} X_{i} \backslash X_{i}\right)=\bigcup\left\{X_{j} \mid j \supsetneqq i\right\},
$$

we suppose there is a closed neighborhood $N_{i}$ of $\Sigma X_{i}$ in $X^{i}=\operatorname{cl} X_{i}$ and a map $\nu_{i}: \partial N_{i} \rightarrow \Sigma X_{i}$ such that:

1. $\partial N_{i}$ is a codimension- 1 submanifold of $X_{i}$,

2. $N_{i}$ is the mapping cylinder of $\nu_{i}$ (with $\partial N_{i}$ and $\Sigma X_{i}$ corresponding to the top and bottom of the cylinder),

3. if $j \supsetneqq i$ and $W_{j}=X_{j} \backslash \operatorname{int} N_{j}$, then $\left.\nu_{i}\right|_{\nu_{i}^{-1}\left(W_{j}\right)}: \nu_{i}^{-1}\left(W_{j}\right) \rightarrow W_{j}$ is a submersion (in the appropriate category).

(See Fig. 2). Such mapping cylinder neighborhoods do not always exist in the

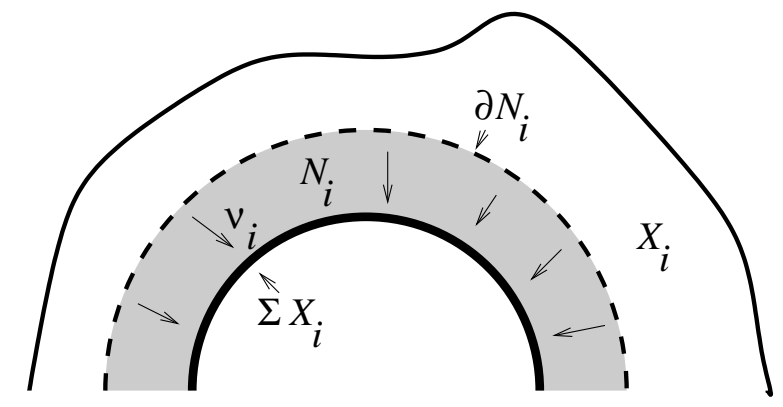

Fig. 2. A mapping cylinder neighborhood

weakest types of stratified sets, but an obstruction theory for their existence was given in [72, Theorem 1.7].

In the PL Browder-Quinn theory, Whitehead torsion and the $s$-cobordism theorem take an especially nice form. The appropriate obstruction group for 
a PL stratified space $X$ with strata $X_{i}$ as above is simply

$$
\mathrm{Wh}^{\mathrm{BQ}}(X)=\bigoplus_{i} \mathrm{Wh}\left(\operatorname{cl} X_{i}\right) .
$$

An $h$-cobordism $W$ of stratified spaces, based on $X$, is itself a stratified space with boundary $X \amalg X^{\prime}$, where the inclusions of $X$ and $X^{\prime}$ into $W$ are stratified homotopy equivalences, and the neighborhood data for the strata of $Z$ are the pullbacks with respect to the retractions of the data for $X$ (and of $X^{\prime}$ ).

Theorem 5.3 (Stratified $s$-cobordism theorem $[103, \S 6]$ ). Let $X$ be a $P L$ stratified space in the sense of Browder-Quinn above. Then assuming all strata have dimension $\geq 5, P L h$-cobordisms of $P L$ stratified spaces based on $X$ are in natural bijection with $\mathrm{Wh}^{\mathrm{BQ}}(X)$.

One thing to keep in mind, however, is that in the stratified (or equivariant) world, the parallelism between the three categories of topological, PL, and smooth manifolds breaks down. The stratified topological $s$-cobordism theorem is quite different from the PL one, and involves a rather different obstruction group $\mathrm{Wh}^{\text {top }}(X)$. One can already see this in the case of the one-point compactification $X=M_{+}$of a non-compact manifold $M$, say with $M$ PL (or even smooth). The space $X$ has two strata, $M$ and a point, so $\mathrm{Wh}^{\mathrm{BQ}}(X)=\mathrm{Wh}\left(\pi_{1}(X)\right)$, whereas $\mathrm{Wh}^{\text {top }}(X)=\mathrm{Wh}^{p}(M)$, the proper Whitehead group that appears in Theorem 5.1 (see [103, pp. 131-132] for an explanation of why this is the case). Also note that since $\mathrm{Wh}^{\text {top }}(X)$ is a kind of "relative" Whitehead group, it can involve $K_{0}$ and lower $K$-groups of the strata, not just Whitehead groups of the closed strata as in the case of $\mathrm{Wh}^{\mathrm{BQ}}(X)$.

\section{Waldhausen's $A$-Theory}

For some of the applications of $K$-theory to geometric topology, one needs a variant of algebraic $K$-theory called the algebraic $K$-theory of spaces, $A$ theory, or Waldhausen $K$-theory. There are several equivalent versions of the definition of Waldhausen's $A(X)$, but all of them are somewhat involved. So it's worth giving the informal definition first. If $X$ is a pointed space, let $Q\left((\Omega X)_{+}\right)=\Omega^{\infty} \Sigma^{\infty}\left((\Omega X)_{+}\right)$be the infinite loop space whose homotopy groups are the stable homotopy groups of $(\Omega X)_{+}$, the loop group of $X$ with a disjoint basepoint attached. The space $Q\left((\Omega X)_{+}\right)$can be viewed as a "ring up to homotopy," the multiplication coming from concatenation of loops in $\Omega X$. If $X$ is path-connected, then $\pi_{0}(\Omega X)=\pi_{1}(X)$ is an actual group, and there is a map $Q\left((\Omega X)_{+}\right) \rightarrow \mathbb{Z} \pi_{1}(X)$ from $Q\left((\Omega X)_{+}\right)$to a genuine ring, the group ring of $\pi_{1}(X)$. (The map $Q\left(*_{+}\right)=Q\left(S^{0}\right) \rightarrow \mathbb{Z}$ sends a stable map $S^{0} \rightarrow S^{0}$, i.e., a map $S^{n} \rightarrow S^{n}$ for some $n$, to its degree.) Waldhausen's $A(X)$ [93] is the $K$-theory space (the space whose homotopy groups are the $K$ groups) of the ring up to homotopy $Q\left((\Omega X)_{+}\right)$, and the map $Q\left((\Omega X)_{+}\right) \rightarrow$ $\mathbb{Z} \pi_{1}(X)$ induces a "linearization map" $L: A(X) \rightarrow \mathbb{K}\left(\mathbb{Z} \pi_{1}(X)\right)$ which is close 
to being an equivalence in "low degrees." More precisely, the space $A(X)$ splits as $Q\left(X_{+}\right) \times \mathbf{W h}^{\mathrm{diff}}(X)$ ([94], [95], and [99]) for a certain homotopy functor $\mathbf{W h}^{\text {diff }}$ to be discussed further in Section 7 below, but related to the (higher) Whitehead groups of $\pi_{1}(X)$. The homotopy fiber of

$$
L: A(*) \rightarrow \mathbb{K}(\mathbb{Z})=\mathbb{Z} \times B G L(\mathbb{Z})^{+}
$$

has finite homotopy groups, and localized at a prime $p$ is known to be $(2 p-3)$ connected, with its first homotopy group isomorphic to $\mathbb{Z} / p$ in degree $2 p-2$ ([97], [55, Theorem 1.2]).

The main foundational paper on $A(X)$, giving a rigorous definition and proving the key properties, is [98]. As this is a 100-page technical tour de force, there is no hope to explain it all here, so we will just quickly summarize some of the key points. The longest part of the paper explains a method for defining the $K$-theory of a category with cofibrations and weak equivalences. Such a category $\mathcal{C}$ has a zero object and satisfies certain axioms modeled on the properties of the category of finite pointed simplicial sets, where the cofibrations and weak equivalences are defined as usual in homotopy theory. Other examples of this structure are exact categories in the sense of Quillen, with the admissible monomorphisms as cofibrations and the isomorphisms as weak equivalences.

Given a category $\mathcal{C}$ with cofibrations and weak equivalences, Waldhausen introduces the simplicial category $w \mathcal{S}_{\bullet} \mathcal{C}$. The category $w \mathcal{S}_{n} \mathcal{C}$ in degree $n$ of this simplicial category has as its objects the diagrams

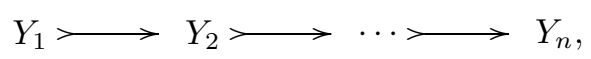

with the arrows $\longrightarrow$ denoting cofibrations, and as its morphisms the diagrams

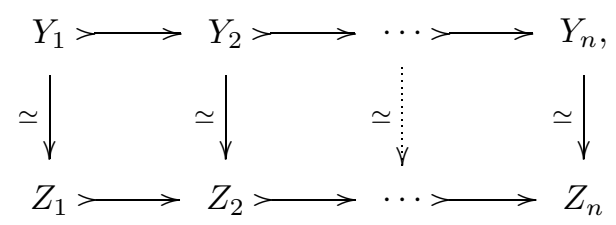

with the vertical arrows weak equivalences. One also needs to specific choices of quotients $Y_{j} / Y_{i}$. Thus, for example, $w \mathcal{S}_{0} \mathcal{C}$ is the trivial category consisting only of the 0 -object and the 0 -morphism, and $w \mathcal{S}_{1} \mathcal{C}$ is (equivalent to) the category $w \mathcal{C}$ of weak equivalences in $\mathcal{C}$. The $K$-theory $\mathbb{K}(\mathcal{C})$ of the category $\mathcal{C}$ can then be defined to be $\Omega\left|w \mathcal{S}_{\bullet} \mathcal{C}\right|$. This turns out to be an infinite loop space [98, p. 342]. Also, Waldhausen shows that this definition is essentially equivalent to the usual definition (via the + -construction or $Q$-construction) of Quillen $K$-theory (when both make sense). The equivalence $w \mathcal{C} \stackrel{\simeq}{\rightarrow} w \mathcal{S}_{1} \mathcal{C}$ gives rise to a map $\Sigma|w \mathcal{C}| \hookrightarrow|w \mathcal{S} . \mathcal{C}|$, and thus to a dual map 


$$
|w \mathcal{C}| \rightarrow \Omega\left|w \mathcal{S}_{\bullet} \mathcal{C}\right|=_{\text {def }} \mathbb{K}(\mathcal{C}) .
$$

The algebraic $K$-theory of a space $X$ (which we think of as a simplicial setpassage from simplicial sets to spaces is given by the geometric realization functor $|\cdot|)$ is then defined to be $\mathbb{K}\left(\mathcal{R}_{f}(X)\right)$, where $\mathcal{R}_{f}(X)$ is the category of finite retractive spaces over $X$, or in other words, simplicial sets $Y$ equipped with an inclusion $X \hookrightarrow Y$, plus a retraction $r: Y \rightarrow X$, so that $Y$ consists of the union of $X$ and finitely many additional simplices. The map $X \mapsto A(X)$ is a homotopy functor of $X$ [98, Proposition 2.1.7], and there is a pairing $A(X) \wedge A\left(X^{\prime}\right) \rightarrow A\left(X \times X^{\prime}\right)\left[96\right.$, pp. 400-402]. The map $\left|w \mathcal{R}_{f}(*)\right| \rightarrow A(*)$ is characterized by a certain additivity property [96, Lemmas 1.1 and 1.2]; on the level of $\pi_{0}$, it sends a homotopy equivalence class of finite spaces (or simplicial sets) to $\pi_{0}(A(*))=\mathbb{Z}$, and turns out to be the Euler characteristic. Other applications of the algebraic $K$-theory of spaces will be mentioned in the following section, Section 7. But we just mention that $A(X)$ satisfies an analogue of the "fundamental theorem of $K$-theory" (the calculation of $K_{*}\left(R\left[t, t^{-1}\right]\right)$ in terms of $\left.K_{*}(R)\right)$ :

Theorem 6.1 ([44], [45], [77]). There is a splitting of $A\left(X \times S^{1}\right)$ as

$$
A\left(X \times S^{1}\right) \simeq A(X) \times \Omega^{-1} A(X) \times \text { "Nil term" } \times \text { "Nil term". }
$$

(This notation isn't completely precise but is meant to imply that the second factor is a delooping of $A(X)$. More details may be found in the original papers.) The two Nil terms are homeomorphic, and the "canonical involution" on $A\left(X \times S^{1}\right)$ (analogous to the involution on $K$-theory of rings coming from the conjugate transpose on matrices) interchanges the two Nil terms and restricts to the canonical involutions on the other two factors.

Given that the definition of $A$-theory involves so much abstract machinery, it is perhaps surprising that so much is known about how to calculate $A(X)$. One of the key tools in this regard is the cyclotomic trace of Bökstedt, Hsiang, and Madsen [8], a functorial map Trc: $A(X) \rightarrow T C(X ; p)$ from $A$-theory to topological cyclic homology. (To define this map, it is necessary to first fix a prime $p$.) There is a beautiful theorem of Dundas about the fiber of this map after $p$-completion:

Theorem 6.2 (Dundas [28]). If $X$ is connected, then the diagram

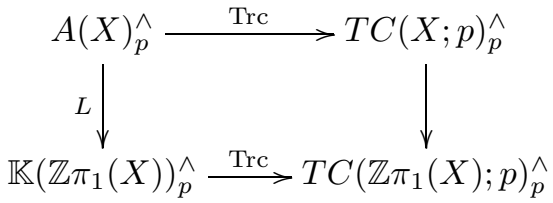

is homotopy Cartesian (i.e., is a homotopy pullback square).

In particular, the fiber of the cyclotomic trace map (after $p$-completion) only depends on $\pi_{1}(X)$, and not on the rest of the homotopy type of $X$. 
(This was earlier proved in [7].) And after $p$-completion, the homotopy fiber of the linearization map from $A$-theory to $K$-theory of the group ring can be computed entirely from $T C$-theory.

\section{$7 K$-Theory and Pseudo-Isotopy}

Let $M$ be a compact smooth manifold (for now without boundary, but we will be forced to consider manifolds with boundary later). The space of pseudoisotopies (or concordances) of $M$ is defined to be

$$
\mathcal{C}(M)=\operatorname{Diff}(M \times I \text { rel }(M \times\{0\} \cup \partial M \times I)),
$$

with the $C^{\infty}$ topology. (See Fig. 3.) This is of course a topological group under composition of diffeomorphisms. A basic problem in manifold topology

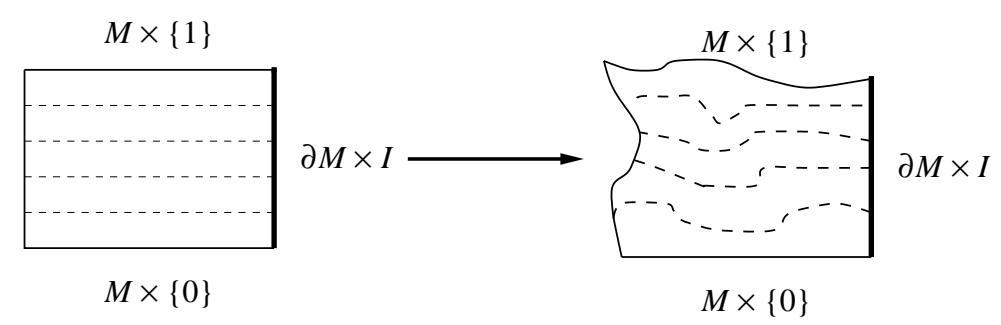

Fig. 3. A pseudo-isotopy

is to understand this space, and especially its set of connected components. This problem is closely related to computing $\pi_{0}(\operatorname{Diff}(M))$, the group of diffeomorphisms of $M$ modulo isotopy. The reason is that, on the one hand, an isotopy of diffeomorphisms of $M$ clearly induces a pseudo-isotopy. But not every pseudo-isotopy comes from an isotopy, since the "level sets" $M \times\{t\}$ don't have to be preserved for $t>0$. (Again see Fig. 3.) But $\mathcal{C}(M)$ acts continuously on $\operatorname{Diff}(M)$ by $h \cdot g=\left.h\right|_{M \times\{1\}} g$, and the (open) orbit of the identity is the group of diffeomorphisms pseudo-isotopic to the identity. So if $\mathcal{C}(M)$ is path-connected, pseudo-isotopic diffeomorphisms are isotopic. The first major result about $\pi_{0}(\mathcal{C}(M))$ was a difficult theorem of Cerf [18]: $\mathcal{C}(M)$ is path-connected if $M$ is simply connected and $\operatorname{dim} M \geq 6 .{ }^{5}$ However, it was soon discovered that even in high dimensions, $\mathcal{C}(M)$ can be disconnected if $\pi_{1}(M)$ is non-trivial, and Hatcher and Wagoner [37] (originally working independently) discovered a remarkable connection between $\pi_{0}(\mathcal{C}(M))$ and the $K$-group $K_{2}\left(\mathbb{Z} \pi_{1}(M)\right)$. This eventually led to an exact sequence for $\pi_{0}(\mathcal{C}(M))$ :

\footnotetext{
${ }^{5}$ There is an obvious analogue of $\mathcal{C}(M)$, denoted $\mathcal{C}^{\mathrm{PL}}(M)$, for PL manifolds, where we replace Diff by the group of PL automorphisms. The analogue of Cerf's theorem holds for this as well [75].
} 


$$
\begin{aligned}
K_{3}\left(\mathbb{Z} \pi_{1}(M)\right) \rightarrow \mathrm{Wh}_{1}^{+}\left(\pi_{1}(M) ; \mathbb{Z} / 2\right. & \left.\times \pi_{2}(M)\right) \\
& \rightarrow \pi_{0}(\mathcal{C}(M)) \rightarrow \mathrm{Wh}_{2}\left(\pi_{1}(M)\right) \rightarrow 0 .
\end{aligned}
$$

Here $\mathrm{Wh}_{2}\left(\pi_{1}(M)\right)$ denotes the quotient of $K_{2}\left(\mathbb{Z} \pi_{1}(M)\right)$ by its intersection (when we think of $K_{2}$ as a subgroup of the Steinberg group) with the subgroup of the Steinberg group $\operatorname{St}\left(\mathbb{Z} \pi_{1}(M)\right)$ generated by the special elements $w_{i j}(g)$, $g \in \pi_{1}(M)$. This insures that we divide $K_{2}$ by its trivial part (the image of $K_{2}(\mathbb{Z}) \cong \mathbb{Z} / 2$ ). (See [74, Definition 4.4.25].) The second term in (5) is to be interpreted using the definition

$$
\mathrm{Wh}_{1}^{+}(\pi ; A)=H_{0}(\pi, A \pi) / H_{0}(\pi, A) .
$$

Note that we need to keep track of the action of $\pi_{1}(M)$ on $\pi_{2}(M)$ to compute this. Hatcher and Wagoner [37] constructed the surjection $\pi_{0}(\mathcal{C}(M)) \rightarrow$ $\mathrm{Wh}_{2}\left(\pi_{1}(M)\right)$ in (5), Hatcher [37] extended the exact sequence to $\mathrm{Wh}_{1}^{+}$, and K. Igusa [47] corrected a mistake of Hatcher and extended the sequence to $K_{3}$.

The exact sequence (5), along with Igusa's work in [47] showing how the first Postnikov invariant $k_{1}(M) \in H^{3}\left(\pi_{1}(M), \pi_{2}(M)\right)$ can affect $\pi_{0}(\mathcal{C}(M))$, makes it clear that calculation of the topology of $\mathcal{C}(M)$ must in general be quite complicated. Since this problem is hard and "unstable," it is useful to "stabilize." One can define a suspension map $\sigma: \mathcal{C}(M) \rightarrow \mathcal{C}(M \times I)$. (The subtlety here is that if $M$ has a boundary, $M \times I$ is a manifold with corners, but still, there is no problem in suspending a pseudo-isotopy $\varphi$ to $\varphi \times \mathrm{id}_{I}$.) The inductive limit $\mathcal{P}(M)=\lim \mathcal{C}\left(M \times I^{n}\right)$ turns out to be an infinite loop space whose structure can be calculated in many cases; more about this later. Then one can obtain results about $\mathcal{C}(M)$ itself thanks to a second result of Igusa (quite technical to prove):

Theorem 7.1 ([48]). The suspension map $\sigma: \mathcal{C}(M) \rightarrow \mathcal{C}(M \times I)$ is $k$ connected if $\operatorname{dim} M \geq \max (2 k+7,3 k+4)$.

Igusa's proof follows an outline in [35] of an analogous theorem for $\mathcal{C}^{\mathrm{PL}}$ of a PL manifold, but there are problems with the PL proof given there, due to the fact that pushouts do not exist for most pairs of maps of polyhedra. However, concordance stability for smooth manifolds implies stability for PL or topological concordances, for manifolds that have a smooth structure, by a result of Burghelea and Lashof [13].

Before proceeding to the more technical aspects of pseudo-isotopy, it might be worth explaining the rough idea of why $\pi_{0}(\mathcal{C}(M))$ is related to $K_{2}$ (and in fact surjects onto $\left.\mathrm{Wh}_{2}\left(\pi_{1}(M)\right)\right)$. The ideas here come from the papers of Cerf [18] and Hatcher-Wagoner [37] quoted above. The starting point of the proof is an observation of Cerf that $\mathcal{C}(M)$ is homotopy-equivalent to the space $\mathcal{E}(M)$ of functions $f: M \times[0,1] \rightarrow[0,1]$ which are smooth, have no critical points, and satisfy $f(x, 0)=0$ and $f(x, 1)=1$ for all $x \in M$. The homotopy equivalence is simply the map that sends $h \in \mathcal{C}(M)$ to $f:(x, t) \mapsto p_{2} \circ h(x, t)$, where $p_{2}$ : $M \times[0,1] \rightarrow[0,1]$ is projection onto the 2 nd coordinate. A homotopy inverse 
$\mathcal{E}(M) \rightarrow \mathcal{C}(M)$ to this map is constructed by fixing a Riemannian metric on $M$ and sending $f \in \mathcal{E}(M)$ to the pseudo-isotopy constructed from its gradient flow. So given $h \in \mathcal{C}(M)$, its obstruction in $\mathrm{Wh}_{2}(\pi)$ will be constructed using a path $f_{t}$ of smooth functions $M \times[0,1] \rightarrow[0,1]$ with $f_{0}=p_{2}$ and $f_{1}=p_{2} \circ h$. If this path can be deformed to one with no critical points, then $h$ must lie in the identity component of $\mathcal{C}(M)$. One starts by using the usual ideas of differential topology to deform $f$ to a "generic" function with non-degenerate isolated critical points, and then analyzes what happens as one goes from one critical point to the next (so far this is like the start of the proof of the $h$-cobordism theorem). In the simplest case where all the critical points are either of index $i$ or index $i+1$, one gets for each $t$ a realization of $M \times[0,1]$ as being obtained from $M \times[0,1]$ by attaching $i$-handles and $(i+1)$-handles. Since $M \times[0,1]$ is topologically a product, these handles have to cancel as far as their effect on $\left(\pi_{1}(M)\right.$-equivariant) homology of the universal cover is concerned, so one gets an intersection matrix $A(t)$ in $G L\left(\mathbb{Z} \pi_{1}(M)\right)$ measuring how the $i$-handles (coming from critical points of index $i$ ) are cancelled by the $(i+1)$-handles. The function $t \mapsto A(t)$ also has to be piecewise constant, with jumps just at the critical values of $t$. For $t$ close to $0, A(t)$ is the identity matrix; near $t=1$ it is a product of a permutation matrix and a diagonal matrix with entries of the form $\pm g, g \in \pi_{1}(M)$; and in between it changes finitely many times by certain elementary matrices $e_{j k}( \pm g)$. So if one takes the Steinberg generators $x_{j k}( \pm g)$ corresponding to the $e_{j k}( \pm g)$, one finds that their product gives rise to an element of $\operatorname{St}\left(\mathbb{Z} \pi_{1}(M)\right)$ which lifts $A(1)$. However there is a canonical way to lift any product of a permutation matrix and a diagonal matrix, and in particular $A(1)$, as a product of the $w_{j k}( \pm g)$ 's. Dividing, one gets an element of $K_{2}(\mathbb{Z} \pi)$ which is well-defined modulo the subgroup of $\operatorname{St}\left(\mathbb{Z} \pi_{1}(M)\right)$ generated by all $w_{i j}(g), g \in \pi_{1}(M)$, i.e., an element of $\mathrm{Wh}_{2}\left(\pi_{1}(M)\right)$. One can show that this element doesn't change under smooth deformation, so it gives an obstruction to being able to deform $f$ to a function without critical points.

A program for studying the stabilized pseudo-isotopy space $\mathcal{P}^{\mathrm{PL}}(M)$ in the PL category, by relating it to more homotopy-theoretic objects, was sketched in [36] and [35] without rigorous proofs. A vast generalization of the program was developed and carried out by Waldhausen. He introduced homotopy functors $\mathbf{W h}^{\mathrm{PL}}$ and $\mathbf{W} \mathbf{h}^{\text {diff }}$ with the properties that $\Omega^{2} \mathbf{W h}^{\text {diff }}(M) \simeq \mathcal{P}(M)$ for compact smooth manifolds and $\Omega^{2} \mathbf{W h}^{\mathrm{PL}}(M) \simeq \mathcal{P}^{\mathrm{PL}}(M)$ for compact PL manifolds. As we mentioned before (near the beginning of section 6), Waldhausen showed that $\mathbf{W} \mathbf{h}^{\text {diff }}(X)$ is one factor in $A(X)$. (The other factor is $Q\left(X_{+}\right)$.) There is also a map $A(X) \rightarrow \mathbf{W h}^{\mathrm{PL}}(X)$, and its homotopy fiber is a homology theory, but it's a little harder to understand. The correct analogue of the formula (5) for general $X$ is an exact sequence [46, Theorem 13.1]:

$$
\begin{aligned}
\pi_{3}(A(X)) \rightarrow K_{3}\left(\mathbb{Z} \pi_{1}(X)\right) \rightarrow H_{0}\left(\pi_{1}(X)\right. & \left.,\left(\pi_{2}(X) \oplus \mathbb{Z} / 2\right) \pi_{1}(X)\right) \\
& \rightarrow \pi_{2}(A(X)) \rightarrow K_{2}\left(\mathbb{Z} \pi_{1}(X)\right) \rightarrow 0
\end{aligned}
$$


The machinery that's known for computing $A(X)$ (at least rationally) in some circumstances thus implies quite a lot of information about pseudoisotopies and groups of homeomorphisms and diffeomorphisms of manifolds. For example, Farrell and Jones [30, Corollaries 10.6 and 10.7] compute the rational homotopy groups $\pi_{j}(\operatorname{Homeo}(M)) \otimes_{\mathbb{Z}} \mathbb{Q}$ and $\pi_{j}(\operatorname{Diff}(M)) \otimes_{\mathbb{Z}} \mathbb{Q}$ for $M$ a real hyperbolic manifold of dimension $m>10$ and $j$ in a stable range $(\leq(m-4) / 3)$. The connection between $A(X)$ and pseudo-isotopies also makes it possible to study not only "higher" Whitehead torsion (as in [35]), but also higher Reidemeister torsion (as in [49]).

We should point out also that there are controlled versions of pseudoisotopy theory, which are related to negative $K$-theory (e.g., [2], [43], and [41]).

\section{$8 K$-Theory and Symbolic Dynamics}

Among the lesser known applications of $K$-theory to geometric topology are applications to symbolic dynamics, the study of invariant subspaces of the shift map acting on infinite sequences of letters from some alphabet. To fix notation, consider the full $n$-shift, $X_{n}^{+}=\{0,1, \cdots, n-1\}^{\mathbb{N}}$ or $X_{n}=\{0,1, \cdots, n-1\}^{\mathbb{Z}}$ with the product topology. (Topologically, $X_{n}^{+}$and $X_{n}$ are both Cantor sets if $n>1$.) Let $\sigma_{n}: X_{n} \rightarrow X_{n}$ and $\sigma_{n}^{+}: X_{n}^{+} \rightarrow X_{n}^{+}$be the shift map that shifts a sequence one unit to the left. The map $\sigma_{n}$ is a self-homeomorphism of $X_{n}$, called the two-sided n-shift, and $\sigma_{n}^{+}$is a surjective (but non-invertible) self-map of $X_{n}^{+}$, called the one-sided $n$-shift.

A subshift of finite type is a pair $\left(X_{A}, \sigma_{A}\right)$, where $A$ is an $n \times n$ matrix with entries in $\{0,1\}$, where $X_{A}$ is the closed $\sigma_{n}$-invariant subset of $X_{n}$ consisting of sequences $\left(x_{k}\right)$ with allowable transitions, i.e., with $A_{x_{k}, x_{k+1}}=1$ for all $k$, and where $\sigma_{A}=\left.\sigma_{n}\right|_{X_{A}}$. The one-sided subshift of finite type $\left(X_{A}^{+}, \sigma_{A}^{+}\right)$is defined similarly from $\left(X_{n}^{+}, \sigma_{n}^{+}\right)$. The first basic problem of symbolic dynamics is to classify the pairs $\left(X_{A}, \sigma_{A}\right)$ and $\left(X_{A}^{+}, \sigma_{A}^{+}\right)$up to topological conjugacy. Note that keeping track of the shift structure is essential here, since all $X_{A}$ 's and $X_{A}^{+}$'s are Cantor sets ${ }^{6}$ and are thus homeomorphic to one another, regardless of the values of $n$ and of the matrix $A$.

One might, of course, wonder why we are considering homeomorphisms of Cantor sets when we promised at the beginning of this article to restrict attention to "topology of manifolds and manifold-like spaces, of simplicial and CW-complexes, and of automorphisms of such objects." The reason is that as amply demonstrated by Smale [80], Bowen [10], and others, any attempt to study the dynamics of smooth self-maps of manifolds inevitably leads to problems of symbolic dynamics.

For purposes of studying the conjugacy problem for the pairs $\left(X_{A}, \sigma_{A}\right)$, it's convenient to allow $A$ to be any square matrix with entries in $\mathbb{N}$, the

\footnotetext{
${ }^{6}$ This is assuming we are not in one of the uninteresting cases where $X_{A}$ or $X_{A}^{+}$ contains an isolated point, as when $A=(1)$.
} 
non-negative integers. There is a canonical way to do this [91, pp. 272-273] without changing the definition of $X_{A}$ in the case of a $0-1$ matrix, and so that the $1 \times 1$ matrix $(n)$ and the $n \times n$ matrix with all entries equal to 1 both give rise to $X_{n}$. However, any $X_{A}$ can be rewritten as $X_{A^{\#}}$ for some 0-1 matrix $A^{\#}$ (usually of larger size than $A$ ).

The key initial work on the conjugacy problem for the pairs $\left(X_{A}, \sigma_{A}\right)$ was done by Williams [104], who showed that $\sigma_{A}$ and $\sigma_{B}$ are topologically conjugate if there are rectangular (not necessarily square!) matrices $R$ and $S$ with entries in $\mathbb{N}$ such that $R S=A, S R=B$. This relation is called elementary strong shift equivalence over $\mathbb{N}$, but this is a slight misnomer: it is not an equivalence relation. The equivalence relation it generates (on square matrices of arbitrary size with entries in $\mathbb{N}$ ) is called strong shift equivalence over $\mathbb{N}$, and Williams proved that $\sigma_{A}$ and $\sigma_{B}$ are topologically conjugate if and only if the matrices $A$ and $B$ are strong shift equivalent over $\mathbb{N}$. Williams also gave a necessary and sufficient condition for topological conjugacy of the one-sided shifts $\sigma_{A}^{+}$and $\sigma_{B}^{+}$in terms of conjugacy of "total amalgamations," and this criterion is computable. However, strong shift equivalence is not especially computable - the problem is that there is no obvious way to bound the length of a chain of elementary strong shift equivalences. Thus Williams also introduced another equivalence relation. Two square matrices $A$ and $B$ with entries in $\mathbb{N}$ are called shift equivalent over $\mathbb{N}$ if there are rectangular matrices $R$ and $S$ with entries in $\mathbb{N}$ such that $A R=R B, S A=B S$, and for some $k \geq 1, A^{k}=R S$ and $B^{k}=S R$. It turns out that shift equivalence over $\mathbb{N}$ is computable and that the matrices $A$ and $B$ are shift equivalent over $\mathbb{N}$ if and only if $\sigma_{A}^{k}$ and $\sigma_{B}^{k}$ are topologically conjugate for all sufficiently large $k$. An unsolved problem for many years, called the shift equivalence problem, was whether shift equivalence implies strong shift equivalence (over $\mathbb{N}$ ), or equivalently, if conjugacy of $\sigma_{A}^{k}$ and $\sigma_{B}^{k}$ for all large $k$ implies conjugacy of $\sigma_{A}$ and $\sigma_{B}$.

The (negative) solution to the shift equivalence problem heavily involves $K$-theory. First of all, shift equivalence turns out to be connected with the ordering on $K_{0}$ of a ring, a certain $C^{*}$-algebra associated to the shift. As a result, one can for example prove:

Theorem 8.1 ([91, Corollary 2.13]). If $A, B \in G L(n, \mathbb{Z}) \cap M_{n}(\mathbb{N})$, then $A$ and $B$ are shift equivalent over $\mathbb{N}$ if and only if $A$ and $B$ are conjugate in $G L(n, \mathbb{Z})$.

Also, if one drops the requirement that the matrices defining a shift equivalence have non-negative entries and thus considers shift equivalence and strong shift equivalence over $\mathbb{Z}$, then these two conditions are indeed equivalent [90].

However, over $\mathbb{N}$, Kim and Roush [53] showed that shift equivalence and strong shift equivalence are not equivalent, even for primitive matrices (the most important case). While their original construction did not directly involve $K$-theory, it was partially motivated by work of Wagoner [89] relating $\operatorname{Aut}\left(\sigma_{A}\right)$ to $K_{2}$, and in [92], Wagoner, Kim, and Roush showed that one can 
indeed construct a counterexample to the shift equivalence problem using an invariant based on $K_{2}\left(\mathbb{Z}[t] /\left(t^{2}\right)\right)$. A good introduction to this work may be found in [91]. If one looks careful, one can see the connection with the ideas of Cerf theory and the connection between pseudo-isotopy and $K_{2}$.

\section{References}

1. Douglas R. Anderson. Torsion invariants and actions of finite groups. Michigan Math. J., 29(1):27-42, 1982.

2. Douglas R. Anderson and W. C. Hsiang. The functors $K_{-1}$ and pseudoisotopies of polyhedra. Ann. of Math. (2), 105(2):201-223, 1977.

3. Paweł Andrzejewski. Equivariant finiteness obstruction and its geometric applications - a survey. In Algebraic topology, Poznan 1989, volume 1474 of Lecture Notes in Math., pages 20-37. Springer, Berlin, 1991.

4. Shôrô Araki and Katsuo Kawakubo. Equivariant $s$-cobordism theorems. $J$. Math. Soc. Japan, 40(2):349-367, 1988.

5. Amir Assadi and William Browder. On the existence and classification of extensions of actions on submanifolds of disks and spheres. Trans. Amer. Math. Soc., 291(2):487-502, 1985.

6. Jenny A. Baglivo. An equivariant Wall obstruction theory. Trans. Amer. Math. Soc., 256:305-324, 1979.

7. M. Bökstedt, G. Carlsson, R. Cohen, T. Goodwillie, W. C. Hsiang, and I. Madsen. On the algebraic $K$-theory of simply connected spaces. Duke Math. J., 84(3):541-563, 1996.

8. M. Bökstedt, W. C. Hsiang, and I. Madsen. The cyclotomic trace and algebraic K-theory of spaces. Invent. Math., 111(3):465-539, 1993.

9. Karol Borsuk. On the topology of retracts. Ann. of Math. (2), 48:1082-1094, 1947.

10. Rufus Bowen. Markov partitions for Axiom A diffeomorphisms. Amer. J. Math., 92:725-747, 1970 .

11. William Browder and Frank Quinn. A surgery theory for $G$-manifolds and stratified sets. In Manifolds - Tokyo 1973 (Proc. Internat. Conf., Tokyo, 1973), pages 27-36. Univ. Tokyo Press, Tokyo, 1975.

12. D. Burghelea, L. Friedlander, T. Kappeler, and P. McDonald. Analytic and Reidemeister torsion for representations in finite type Hilbert modules. Geom. Funct. Anal., 6(5):751-859, 1996.

13. D. Burghelea and R. Lashof. Stability of concordances and the suspension homomorphism. Ann. of Math. (2), 105(3):449-472, 1977.

14. Sylvain Cappell and Shmuel Weinberger. Homology propagation of group actions. Comm. Pure Appl. Math., 40(6):723-744, 1987.

15. Sylvain Cappell and Shmuel Weinberger. A simple construction of AtiyahSinger classes and piecewise linear transformation groups. J. Differential Geom., 33(3):731-742, 1991.

16. Gunnar Carlsson. Bounded $K$-theory and the assembly map in algebraic $K$ theory. In Novikov conjectures, index theorems and rigidity, Vol. 2 (Oberwolfach, 1993), volume 227 of London Math. Soc. Lecture Note Ser., pages 5-127. Cambridge Univ. Press, Cambridge, 1995. 
17. Henri Cartan and Samuel Eilenberg. Homological algebra. Princeton Landmarks in Mathematics. Princeton University Press, Princeton, NJ, 1999. With an appendix by David A. Buchsbaum, Reprint of the 1956 original.

18. Jean Cerf. La stratification naturelle des espaces de fonctions différentiables réelles et le théorème de la pseudo-isotopie. Inst. Hautes Études Sci. Publ. Math., (39):5-173, 1970.

19. T. A. Chapman. Topological classification of simple homotopy equivalences. In Proceedings of the International Congress of Mathematicians (Vancouver, B. C., 1974), Vol. 1, pages 509-513. Canad. Math. Congress, Montreal, Que., 1975 .

20. T. A. Chapman. Controlled simple homotopy theory and applications, volume 1009 of Lecture Notes in Mathematics. Springer-Verlag, Berlin, 1983.

21. T. A. Chapman and Steve Ferry. Approximating homotopy equivalences by homeomorphisms. Amer. J. Math., 101(3):583-607, 1979.

22. Jeff Cheeger. Analytic torsion and the heat equation. Ann. of Math. (2), 109(2):259-322, 1979 .

23. Shiing Shen Chern and James Simons. Some cohomology classes in principal fiber bundles and their application to riemannian geometry. Proc. Nat. Acad. Sci. U.S.A., 68:791-794, 1971.

24. Shiing Shen Chern and James Simons. Characteristic forms and geometric invariants. Ann. of Math. (2), 99:48-69, 1974.

25. Marshall M. Cohen. A course in simple-homotopy theory. Springer-Verlag, New York, 1973. Graduate Texts in Mathematics, Vol. 10.

26. J. F. Davis and R. J. Milgram. A survey of the spherical space form problem, volume 2 of Mathematical Reports. Harwood Academic Publishers, Chur, 1985.

27. Pierre Deligne and Dennis Sullivan. Fibrés vectoriels complexes à groupe structural discret. C. R. Acad. Sci. Paris Sér. A-B, 281(24):Ai, A1081-A1083, 1975.

28. Bjørn Ian Dundas. Relative $K$-theory and topological cyclic homology. Acta Math., 179(2):223-242, 1997.

29. Johan L. Dupont. Curvature and characteristic classes. Springer-Verlag, Berlin, 1978. Lecture Notes in Mathematics, Vol. 640.

30. F. T. Farrell and L. E. Jones. A topological analogue of Mostow's rigidity theorem. J. Amer. Math. Soc., 2(2):257-370, 1989.

31. F. T. Farrell and J. B. Wagoner. Algebraic torsion for infinite simple homotopy types. Comment. Math. Helv., 47:502-513, 1972.

32. Steve Ferry. Homotoping $\varepsilon$-maps to homeomorphisms. Amer. J. Math., 101(3):567-582, 1979.

33. Steve Ferry and Andrew Ranicki. A survey of Wall's finiteness obstruction. In Surveys on surgery theory, Vol. 2, pages 63-79. Princeton Univ. Press, Princeton, NJ, 2001.

34. Steven C. Ferry and Erik K. Pedersen. Epsilon surgery theory. In Novikov conjectures, index theorems and rigidity, Vol. 2 (Oberwolfach, 1993), volume 227 of London Math. Soc. Lecture Note Ser., pages 167-226. Cambridge Univ. Press, Cambridge, 1995.

35. A. E. Hatcher. Higher simple homotopy theory. Ann. of Math. (2), 102(1):101$137,1975$.

36. A. E. Hatcher. Concordance spaces, higher simple-homotopy theory, and applications. In Algebraic and geometric topology (Proc. Sympos. Pure Math., Stanford Univ., Stanford, Calif., 1976), Part 1, Proc. Sympos. Pure Math., XXXII, pages 3-21. Amer. Math. Soc., Providence, R.I., 1978. 
37. Allen Hatcher and John Wagoner. Pseudo-isotopies of compact manifolds. Société Mathématique de France, Paris, 1973. With English and French prefaces, Astérisque, No. 6.

38. Henning Hauschild. Äquivariante Whiteheadtorsion. Manuscripta Math., 26(12):63-82, 1978/79.

39. Jean-Claude Hausmann. Algebraic $K$-theory and flat manifolds. In Algebraic topology, Aarhus 1978 (Proc. Sympos., Univ. Aarhus, Aarhus, 1978), volume 763 of Lecture Notes in Math., pages 212-234. Springer, Berlin, 1979.

40. Jean-Claude Hausmann and Pierre Vogel. The plus construction and lifting maps from manifolds. In Algebraic and geometric topology (Proc. Sympos. Pure Math., Stanford Univ., Stanford, Calif., 1976), Part 1, Proc. Sympos. Pure Math., XXXII, pages 67-76. Amer. Math. Soc., Providence, R.I., 1978.

41. Bruce Hughes and Stratos Prassidis. Control and relaxation over the circle. Mem. Amer. Math. Soc., 145(691):x+96, 2000.

42. Bruce Hughes and Shmuel Weinberger. Surgery and stratified spaces. In Surveys on surgery theory, Vol. 2, volume 149 of Ann. of Math. Stud., pages 319352. Princeton Univ. Press, Princeton, NJ, 2001.

43. C. Bruce Hughes. Bounded homotopy equivalences of Hilbert cube manifolds. Trans. Amer. Math. Soc., 287(2):621-643, 1985.

44. Thomas Hüttemann, John R. Klein, Wolrad Vogell, Friedhelm Waldhausen, and Bruce Williams. The "fundamental theorem" for the algebraic $K$-theory of spaces. I. J. Pure Appl. Algebra, 160(1):21-52, 2001.

45. Thomas Hüttemann, John R. Klein, Wolrad Vogell, Friedhelm Waldhausen, and Bruce Williams. The "fundamental theorem" for the algebraic $K$-theory of spaces. II. The canonical involution. J. Pure Appl. Algebra, 167(1):53-82, 2002.

46. Kiyoshi Igusa. On the algebraic $K$-theory of $A_{\infty}$-ring spaces. In Algebraic $K$ theory, Part II lpOberwolfach, 1980), volume 967 of Lecture Notes in Math., pages 146-194. Springer, Berlin, 1982.

47. Kiyoshi Igusa. What happens to Hatcher and Wagoner's formulas for $\pi_{0} \mathcal{C}(M)$ when the first Postnikov invariant of $M$ is nontrivial? In Algebraic K-theory, number theory, geometry and analysis (Bielefeld, 1982), volume 1046 of Lecture Notes in Math., pages 104-172. Springer, Berlin, 1984.

48. Kiyoshi Igusa. The stability theorem for smooth pseudoisotopies. K-Theory, 2(1-2):vi+355, 1988.

49. Kiyoshi Igusa. Higher Franz-Reidemeister torsion, volume 31 of AMS/IP Studies in Advanced Mathematics. American Mathematical Society, Providence, RI, 2002.

50. Sören Illman. Whitehead torsion and group actions. Ann. Acad. Sci. Fenn. Ser. A I Math., (588):45, 1974.

51. Sören Illman. Actions of compact Lie groups and the equivariant Whitehead group. Osaka J. Math., 23(4):881-927, 1986.

52. Michel A. Kervaire. Le théorème de Barden-Mazur-Stallings. Comment. Math. Helv., 40:31-42, 1965.

53. K. H. Kim and F. W. Roush. The Williams conjecture is false for irreducible subshifts. Ann. of Math. (2), 149(2):545-558, 1999.

54. Robion C. Kirby and Laurence C. Siebenmann. Foundational essays on topological manifolds, smoothings, and triangulations. Princeton University Press, Princeton, N.J., 1977. With notes by John Milnor and Michael Atiyah, Annals of Mathematics Studies, No. 88. 
55. John R. Klein and John Rognes. The fiber of the linearization map $A(*) \rightarrow$ $K(\mathbb{Z})$. Topology, 36(4):829-848, 1997.

56. Wolfgang Lück. The geometric finiteness obstruction. Proc. London Math. Soc. (3), 54(2):367-384, 1987.

57. Wolfgang Lück and Mikael Rørdam. Algebraic $K$-theory of von Neumann algebras. K-Theory, 7(6):517-536, 1993.

58. I. Madsen, C. B. Thomas, and C. T. C. Wall. The topological spherical space form problem. II. Existence of free actions. Topology, 15(4):375-382, 1976.

59. J. P. May. A concise course in algebraic topology. Chicago Lectures in Mathematics. University of Chicago Press, Chicago, IL, 1999.

60. John Milnor. Groups which act on $S^{n}$ without fixed points. Amer. J. Math., 79:623-630, 1957.

61. John Milnor. On the existence of a connection with curvature zero. Comment. Math. Helv., 32:215-223, 1958.

62. John Milnor. Lectures on the h-cobordism theorem. Notes by L. Siebenmann and J. Sondow. Princeton University Press, Princeton, N.J., 1965.

63. John Milnor. Whitehead torsion. Bull. Amer. Math. Soc., 72:358-426, 1966.

64. John Milnor and Oscar Burlet. Torsion et type simple d'homotopie. In Essays on Topology and Related Topics (Mémoires dédiés à Georges de Rham), pages 12-17. Springer, New York, 1970.

65. Werner Müller. Analytic torsion and $R$-torsion of Riemannian manifolds. $A d v$. in Math., 28(3):233-305, 1978.

66. Robert Oliver. Whitehead groups of finite groups, volume 132 of London Mathematical Society Lecture Note Series. Cambridge University Press, Cambridge, 1988.

67. Erik K. Pedersen and Charles A. Weibel. A nonconnective delooping of algebraic K-theory. In Algebraic and geometric topology (New Brunswick, N.J., 1983), volume 1126 of Lecture Notes in Math., pages 166-181. Springer, Berlin, 1985.

68. Erik K. Pedersen and Charles A. Weibel. K-theory homology of spaces. In Algebraic topology (Arcata, CA, 1986), volume 1370 of Lecture Notes in Math., pages 346-361. Springer, Berlin, 1989.

69. Erik Kjaer Pedersen. On the $K_{-i}$-functors. J. Algebra, 90(2):461-475, 1984.

70. Frank Quinn. Ends of maps. I. Ann. of Math. (2), 110(2):275-331, 1979.

71. Frank Quinn. Ends of maps. III. Dimensions 4 and 5. J. Differential Geom., 17(3):503-521, 1982.

72. Frank Quinn. Homotopically stratified sets. J. Amer. Math. Soc., 1(2):441-499, 1988.

73. D. B. Ray and I. M. Singer. $R$-torsion and the Laplacian on Riemannian manifolds. Advances in Math., 7:145-210, 1971.

74. Jonathan Rosenberg. Algebraic K-theory and its applications, volume 147 of Graduate Texts in Mathematics. Springer-Verlag, New York, 1994.

75. C. P. Rourke. Embedded handle theory, concordance and isotopy. In Topology of Manifolds (Proc. Inst., Univ. of Georgia, Athens, Ga., 1969), pages 431-438. Markham, Chicago, Ill., 1970.

76. Colin Patrick Rourke and Brian Joseph Sanderson. Introduction to piecewiselinear topology. Springer Study Edition. Springer-Verlag, Berlin, 1982. Reprint.

77. Roland Schwänzl and Ross E. Staffeldt. The approximation theorem and the K-theory of generalized free products. Trans. Amer. Math. Soc., 347(9):3319$3345,1995$. 
78. L. C. Siebenmann. Finding a boundary for an open manifold of dimension $\geq 5$. Ph.D. Dissertation. Princeton Univ., Princeton, 1965.

79. L. C. Siebenmann. Infinite simple homotopy types. Nederl. Akad. Wetensch. Proc. Ser. A 73 = Indag. Math., 32:479-495, 1970.

80. S. Smale. Differentiable dynamical systems. Bull. Amer. Math. Soc., 73:747$817,1967$.

81. John Smillie. Flat manifolds with non-zero Euler characteristics. Comment. Math. Helv., 52(3):453-455, 1977.

82. P. A. Smith. Stationary points of transformation groups. Proc. Nat. Acad. Sci. U. S. A., 28:293-297, 1942.

83. Mark Steinberger. The equivariant topological s-cobordism theorem. Invent. Math., 91(1):61-104, 1988.

84. Mark Steinberger and James West. Equivariant handles in finite group actions. In Geometry and topology (Athens, Ga., 1985), volume 105 of Lecture Notes in Pure and Appl. Math., pages 277-295. Dekker, New York, 1987.

85. Andrei A. Suslin. Homology of $\mathrm{GL}_{n}$, characteristic classes and Milnor $K-$ theory. In Algebraic K-theory, number theory, geometry and analysis (Bielefeld, 1982), volume 1046 of Lecture Notes in Math., pages 357-375. Springer, Berlin, 1984.

86. Andrei A. Suslin. On the K-theory of local fields. J. Pure Appl. Algebra, 34(2-3):301-318, 1984

87. Richard G. Swan. Periodic resolutions for finite groups. Ann. of Math. (2), $72: 267-291,1960$.

88. C. B. Thomas and C. T. C. Wall. The topological spherical space form problem. I. Compositio Math., 23:101-114, 1971.

89. J. B. Wagoner. Markov partitions and $K_{2}$. Inst. Hautes Études Sci. Publ. Math., (65):91-129, 1987.

90. J. B. Wagoner. Higher-dimensional shift equivalence and strong shift equivalence are the same over the integers. Proc. Amer. Math. Soc., 109(2):527-536, 1990.

91. J. B. Wagoner. Strong shift equivalence theory and the shift equivalence problem. Bull. Amer. Math. Soc. (N.S.), 36(3):271-296, 1999.

92. J. B. Wagoner. Strong shift equivalence and $K_{2}$ of the dual numbers. J. Reine Angew. Math., 521:119-160, 2000. With an appendix by K. H. Kim and F. W. Roush.

93. Friedhelm Waldhausen. Algebraic $K$-theory of topological spaces. I. In $\mathrm{Al}$ gebraic and geometric topology (Proc. Sympos. Pure Math., Stanford Univ., Stanford, Calif., 1976), Part 1, pages 35-60. Amer. Math. Soc., Providence, R.I., 1978.

94. Friedhelm Waldhausen. Algebraic $K$-theory of topological spaces. II. In $\mathrm{Al}$ gebraic topology, Aarhus 1978 (Proc. Sympos., Univ. Aarhus, Aarhus, 1978), pages 356-394. Springer, Berlin, 1979.

95. Friedhelm Waldhausen. Algebraic $K$-theory of spaces, a manifold approach. In Current trends in algebraic topology, Part 1 (London, Ont., 1981), pages 141-184. Amer. Math. Soc., Providence, R.I., 1982.

96. Friedhelm Waldhausen. Operations in the algebraic $K$-theory of spaces. In Algebraic K-theory, Part II (Oberwolfach, 1980), volume 967 of Lecture Notes in Math., pages 390-409. Springer, Berlin, 1982. 
97. Friedhelm Waldhausen. Algebraic $K$-theory of spaces, localization, and the chromatic filtration of stable homotopy. In Algebraic topology, Aarhus 1982 (Aarhus, 1982), pages 173-195. Springer, Berlin, 1984.

98. Friedhelm Waldhausen. Algebraic $K$-theory of spaces. In Algebraic and geometric topology (New Brunswick, N.J., 1983), pages 318-419. Springer, Berlin, 1985.

99. Friedhelm Waldhausen. Algebraic $K$-theory of spaces, concordance, and stable homotopy theory. In Algebraic topology and algebraic K-theory (Princeton, N.J., 1983), pages 392-417. Princeton Univ. Press, Princeton, NJ, 1987.

100. C. T. C. Wall. Finiteness conditions for CW-complexes. Ann. of Math. (2), 81:56-69, 1965.

101. C. T. C. Wall. Finiteness conditions for CW complexes. II. Proc. Roy. Soc. Ser. A, 295:129-139, 1966.

102. Shmuel Weinberger. Constructions of group actions: a survey of some recent developments. In Group actions on manifolds (Boulder, Colo., 1983), volume 36 of Contemp. Math., pages 269-298. Amer. Math. Soc., Providence, RI, 1985.

103. Shmuel Weinberger. The topological classification of stratified spaces. Chicago Lectures in Mathematics. University of Chicago Press, Chicago, IL, 1994.

104. R. F. Williams. Classification of subshifts of finite type. Ann. of Math. (2), 98:120-153; errata, ibid. (2) 99 (1974), 380-381, 1973.

105. Joseph A. Wolf. Spaces of constant curvature. Publish or Perish Inc., Houston, TX, fifth edition, 1984 . 



\section{Index}

+-construction, see plus construction

A-theory, 20-23

Alexander polynomial, 13

algebraic $K$-theory of spaces, 20-23

analytic torsion, 14

category with cofibrations and weak equivalences, 21

Chapman's theorem, 11

Chapman-Ferry Theorem, 15

characteristic classes, 6

secondary, 7

Chern class, 6, 9

Chern-Simons class, 7

Chern-Weil theory, 6

concordance, 23

connection, 5

flat, 5-7

control

bounded, 14

epsilon, 15

controlled $K$-theory, 14

cyclotomic trace, 22

degree of a map, 4, 20

determinant

Kadison-Fuglede, 14

dominated, 2

epsilon, 15

Euler characteristic, 22

Euler class, 6, 7

group action, 16, 17 locally linear, 19

$h$-cobordism, 12

$h$-cobordism theorem, 12

handle decomposition

equivariant, 18

homology sphere, 5

homotopy equivalence

controlled, 15

simple, 10,16

$K$-theory

negative, 14

knot, 13

lens space, $13-14$

linearization map, 20

manifold

parallelizable, 7

mapping cylinder neighborhood, 19

obstruction theory, 3,9

plus construction, 5, 21

Pontrjagin class, 6

principal orbit, 16

propagation

of group actions, 18

pseudo-isotopy, 23

Reidemeister torsion, 13

higher, 26 
$s$-cobordism, 7, 12

$s$-cobordism theorem, 12

controlled, 15

equivariant, 18

proper, 16

stratified, 20

semi-s-cobordism, 7

shift

one-sided, 26

two-sided, 26

shift equivalence, 27

strong, 27

shift equivalence problem, 27

singular set, 16

space

compactly generated, 1

finitely dominated, 2

epsilon, 15

Smith acyclic, 18

stratified, 16, 18

Browder-Quinn, 19-20 spherical space form problem, 3

subshift of finite type, 26

Swan homomorphism, 17

symbolic dynamics, 26

topological cyclic homology, 22

variety

complex algebraic, 16, 19

Waldhausen $K$-theory, 20, 21

Wall finiteness obstruction, 2-4 epsilon-controlled, 15 equivariant, $17-18$

weak equivalence, 1,21

Whitehead group, 11

of finite group, 12

of torsion-free group, 12

Whitehead torsion, 10-12

equivariant, 18

proper, 16

stratified, 19 
